submitted to ApJ

\title{
Parker Instability in a Self-Gravitating Magnetized Gas Disk: I. Linear Stability Analysis
}

\author{
Sang Min Lee ${ }^{1,4}$ and S. S. Hong $g^{2,3,5}$
}

\begin{abstract}
To be a formation mechanism of such large-scale structures as giant molecular clouds (GMCs) and HI superclouds, the classical Parker instability driven by external gravity has to overcome three major obstacles: The convective motion accompanying the instability generates thin sheets than large condensations. The degree of density enhancement achieved by the instability is too low to make dense interstellar clouds. The time and the length scales of the instability are significantly longer and larger than the estimated formation time and the observed mean separation of the GMCs, respectively. This paper examines whether a replacement of the driving agent from the external to the self gravity might remove these obstacles by activating the gravitational instability in the Galactic ISM disk. Self-gravitating, magnetized, gas disk bound by a hot halo medium is subject to a Parker-type instability driven by the self gravity, the usual Jeans instability and the convection. If the disk is thicker than a certain critical value, the Parker-type instability due to perturbations of undular mode assists the Jeans gravitational instability to form large-scale structures. Under the external gravity, the convection triggered by interchange mode perturbations is to grow most rapidly; under the self gravity, however, the Jeans gravitational instability can have a growth rate higher than the limiting growth rate of the convective instability. The self gravity can thus suppress the convective motions, and a cooperative action of the Jeans and the Parker instabilities can remove all the obstacles confronting the classical version of the Parker instability. The mass and mean separation of
\end{abstract}

\footnotetext{
${ }^{1}$ Supercomputing Center, KISTI, Daejeon, 305-600, Korea

${ }^{2}$ Astronomy Program, SEES, Seoul National University, Seoul 151-742, Korea

${ }^{3}$ Department of Infrared Astrophysics, ISAS/JAXA, Sagamihara, Kanagawa, Japan

${ }^{4}$ e-mail: smlee@kisti.re.kr

${ }^{5}$ e-mail: sshong@astroism.snu.ac.kr
} 
the structures resulting from the odd-parity undular mode solution are shown to agree better with the HI superclouds than with the GMCs. We briefly discuss how inclusions of the external gravity and cosmic rays would modify behaviors of the odd-parity undular mode solution.

Subject headings: Instabilities — ISM: giant molecular clouds — ISM: magnetic fields

\section{INTRODUCTION}

In seminal studies of Parker in mid 1960s, he proved that magnetized gas disk under the influence of a constant gravitational acceleration becomes unstable to long wavelength perturbations (Parker 1966, 1967). The instability named after Parker was once thought to be the formation mechanism of giant molecular cloud complexes (GMCs) in the Galaxy (Parker 1966; Mouschovias 1974; Appenzeller 1974; Mouschovias et al. 1974; Blitz \& Shu 1980; Shibata \& Matsumoto 1991; Handa et al. 1992). This notion was based mostly on the results of stability analysis done in two dimensions, where only undular mode can be taken into account. Many subsequent analyses done in three dimensions (eg., Zweibel \& Kulsrud 1975; Hughes \& Proctor 1988), however, called our attention to the interchange mode, which may quickly drive the system convectively unstable. Since the convection grows faster for shorter wavelength perturbations, it tends to shred the Galactic ISM disk into thin sheets of low density before the undular mode of long wavelength perturbations collects enough material to form well-defined large-scale structures near the midplane (Asséo et al. 1978; LachièzeRey et al. 1980). To accept the classical Parker instability as the formation mechanism of such galactic structures as the GMCs or the HI superclouds, one needs means to control the disruptive tendency of the interchange mode. The observed density in GMCs is about a few tens of hydrogen molecules per cubic centimeter. On the other hand, three-dimensional MHD simulations of the Parker instability in uniform and non-uniform external gravities render to the system only a factor 2 enhancement in density over its initial equilibrium value, which is far too low to generate interstellar clouds of even moderate density (Kim et al. 1998; Franco et al. 2002). This low degree of density enhancement is one of the most serious obstacles the Parker instability should overcome to be the GMC formation mechanism.

Under canonical conditions of the Galactic ISM disk (cf., Spitzer 1978), the classical Parker instability driven by a uniform external gravity has the minimum growth time of $\sim 10^{8}$ years and the corresponding length scale $\sim 1.6 \mathrm{kpc}$, which are clearly incompatible with the GMC observations. It was in 1990's that non-uniform models of gravity were tried out as a more realistic driving agent than the uniform one (Giz \& Shu 1993; Kim \& 
Hong 1998). By introducing a non-uniform external gravity, whose acceleration increases linearly with the vertical distance, $z$, from the midplane up to $z \simeq 500 \mathrm{pc}$ and stays constant there on, one may reduce the time and length scales nominally to the degree compatible with the GMCs (Hong \& Kim 1997; Kim \& Hong 1998). However, the reduction of scales was not enough to give candidacy of the GMC formation mechanism back to the classical Parker instability, because the reduced scale values are relevant for activities in the regions of high Galactic altitudes, where vertical acceleration of the chosen external gravity becomes strongest but the GMCs do not generally reside.

The undular mode of perturbations triggers the Parker instability preferably in those disks, where magnetic field strength decreases rapidly with vertical distance, because it is easy for such disk to develop strong magnetic buoyancy (Parker 1955; Hughes \& Proctor 1988). Under the uniform external gravity the field and density decrease with $z$ exponentially; while under the non-uniform external gravity they follow roughly a Gaussian function of $z$. Therefore, it is easier to trigger the Parker instability under the non-uniform gravity than the uniform one. This is how a replacement of the external driving agent from the uniform to the non-uniform gravity has brought to the system a significant reduction in the time scale. However, the reduction of time scale doesn't necessarily mean that dense condensations form near the midplane within a reasonably short period of time. It only means a quick development of diffuse chaotic features high above the central plane.

This line of reasoning led us to replace the driving agent of the Parker instability from the external gravity originated mostly from the Galactic stars to the internal one of the ISM itself. We expect the self gravity will ease the problem of low degree of density enhancement the classical Parker instability faced. The self gravity would prevent the Galactic ISM disk from developing convective activities, while it would also activate the Jeans gravitational instability preferably in the disk midplane. In this paper we will examine time, length and mass scales involved with the Parker and the Jeans instabilities in a magnetized gas disk under the self gravity.

There have been many studies on the effect of magnetic fields upon the Jeans gravitational instability. Chandrasekhar \& Fermi (1953) examined development of the gravitational instability in a uniformly magnetized, plane-parallel gas layer. They showed that the existence of uniform fields doesn't change the critical wavelength for onset of the Jeans instability but only reduces its growth rate. When non-uniform field is considered, the critical wavelength does change and the gas layer becomes more unstable than the uniformly magnetized case (Stodólkeiwicz 1963; Nakano 1988). Nakamura et al. (1991) showed that self-gravitating, magnetized gas disk is subject to the Parker-type and the Jeans instabilities simultaneously. Since all of these investigations were done in the plane defined by the vertical and the mag- 
netic field directions, they couldn't address the question whether the self gravity could put a curb upon flaring motions of the convection. Elmegreen (1982) is the first who seriously examined possible roles of the self gravity in modifying the classical picture of the Parker instability. His stability analysis was, in principle, of the three-dimensional nature. However, he integrated the basic MHD equations over the whole extent of the disk, which made the analysis two-dimensional in reality.

In a more recent study, Nagai et al. (1998) made a fully three-dimensional stability analysis onto a uniformly magnetized, self-gravitating gas disk. However, the Parker instability could not be triggered in their systems, because the magnetic fields were not vertically stratified. Instead they examined how the disk thickness might influence the alignment of filamentary structures with respect to the magnetic field direction. Chou et al. (2000) carried out an extensive analysis on non-uniformly magnetized, self-gravitating gas layers, for some cases including the external gravity as well. Their linear analysis was further extended to nonlinear, three-dimensional MHD simulations. In their systems, however, the interchange mode didn't have a chance to drive convective motions, because only isothermal perturbations were applied to the background equilibrium which was in the same isothermal condition. And contrary to our objectives their main concern was on parsec-scale structures in the interstellar medium. We took adiabatic perturbations in one of our earlier three-dimensional stability analyses and saw a possibility of controlling the convection by the self gravity (Lee \& Hong 1999; Lee et al. 2004).

In principle the Parker instability in the Galactic ISM disk should be studied under simultaneous influence of the external and the self gravities. In this study we take the self gravity as the sole driving agent of the instability and examine what modifications it could bring to the classical picture of the Parker instability. This paper is organized as follows: In $\S 2$ we will construct an initial equilibrium configuration for the self-gravitating, magnetized, isothermal gas disk, and then perform linear stability analysis onto it. In $\S 3$ we will interpret the resulting dispersion relations in terms of the convective, the Parker and the Jeans instabilities. In $\S 4$ we will assess whether introduction of the self gravity as the driving agent might ease the major problems confronting the classical Parker instability. In the same section the resulting scales of the maximum growth rate will be compared with the observations of HI superclouds and GMCs. In the last section we will conclude the paper with some discussions. Appendix A details on coefficients of the linearized perturbation equations, and in Appendix B stable wave modes are isolated. Marginal stability analysis is carried out in Appendix C. 


\section{BASIC FORMULATIONS}

\subsection{Initial Equilibrium Model}

We model the local part of the Galactic ISM disk as a self-gravitating, magnetized, isothermal gas layer, which is initially in magnetohydrostatic equilibrium and bound by a hot halo at $z= \pm z_{\mathrm{a}}$. Unperturbed magnetic field $\vec{B}_{\mathrm{o}}=\left[0, B_{\mathrm{o}}(z), 0\right]$ has azimuthal component only and its strength varies with vertical distance.

In such disk the vertical structure is described by the equation of magnetohydrostatic equilibrium,

$$
\frac{d}{d z}\left[P_{\mathrm{o}}(z)+\frac{B_{\mathrm{o}}(z)^{2}}{8 \pi}\right]=-\rho_{\mathrm{o}}(z) \frac{d \psi_{\mathrm{o}}(z)}{d z},
$$

where $P, \psi$, and $\rho$ denote gas pressure, self-gravitational potential, and gas density, respectively. The potential is to be obtained from the Poisson equation,

$$
\frac{d^{2}}{d z^{2}} \psi_{\mathrm{o}}(z)=4 \pi G \rho_{\mathrm{o}}(z)
$$

and the gas pressure is related to density as

$$
P_{\mathrm{o}}(z)=c_{\mathrm{s}}^{2} \rho_{\mathrm{o}}(z)
$$

with the isothermal sound speed, $c_{\mathrm{s}}$, taken to be equal to the rms velocity of interstellar 'coudlets.' In this study cosmic rays are ignored; instead, we will focus on the role of self gravity. The ratio, $\alpha=B_{\mathrm{o}}^{2}(z) / 8 \pi P_{\mathrm{o}}(z)$, of magnetic to gas pressure is kept constant of $z$ for the initial equilibrium. Equations (1) through (3) then give us the equilibrium stratifications of density and magnetic field

$$
\begin{aligned}
& \rho_{\mathrm{o}}(z)=\rho_{\mathrm{o}}(0) \operatorname{sech}^{2}(z / H), \\
& B_{\mathrm{o}}(z)=B_{\mathrm{o}}(0) \operatorname{sech}(z / H),
\end{aligned}
$$

and the resulting gravitational potential

$$
\psi_{\mathrm{o}}(z)=4 \pi G \rho_{\mathrm{o}}(0) H^{2} \ln [\cosh (z / H)]
$$

where scale height $H$ is defined by

$$
H=c_{\mathrm{s}} \sqrt{\frac{1+\alpha}{2 \pi G \rho_{\mathrm{o}}(0)}} .
$$




\subsection{Linearization}

The linearized MHD equations for a self-gravitating magnetized gas disk are given by

$$
\begin{gathered}
\frac{\partial \rho_{1}}{\partial t}+\nabla \cdot\left(\rho_{\mathrm{o}} \vec{v}\right)=0, \\
\rho_{\mathrm{o}} \frac{\partial \vec{v}}{\partial t}=-\nabla P_{1}+\frac{1}{4 \pi}\left(\nabla \times \vec{B}_{1}\right) \times \vec{B}_{\mathrm{o}}+\frac{1}{4 \pi}\left(\nabla \times \vec{B}_{\mathrm{o}}\right) \times \vec{B}_{1}-\rho_{1} \nabla \psi_{\mathrm{o}}-\rho_{\mathrm{o}} \nabla \psi_{1}, \\
\frac{\partial \vec{B}_{1}}{\partial t}=\nabla \times\left(\vec{v} \times \vec{B}_{\mathrm{o}}\right), \\
\frac{\partial P_{1}}{\partial t}+\gamma P_{\mathrm{o}} \nabla \cdot \vec{v}+(\vec{v} \cdot \nabla) P_{\mathrm{o}}=0,
\end{gathered}
$$

and

$$
\nabla^{2} \psi_{1}=4 \pi G \rho_{1},
$$

where all the symbols have their usual meanings and the ones with subscript 1 denote their perturbed quantities. We take adiabatic relation $P=\kappa \rho^{\gamma}$ as the equation of state for the perturbed material [Eq. (11)].

Seeking solutions of the form, $q_{1}(x, y, z, t)=q_{1}(z) \exp \left[i\left(k_{x} x+k_{y} y-\omega t\right)\right]$, we recast the linearized equations in dimensionless forms as

$$
\begin{gathered}
\Omega \frac{\rho_{1}}{\rho_{\mathrm{o}}}=\xi u_{x}+\eta u_{y}+2 i \Theta u_{z}-i \frac{d u_{z}}{d \zeta}, \\
\Omega u_{x}=\frac{\xi}{1+\alpha} \frac{P_{1}}{P_{\mathrm{o}}}-\frac{2 \alpha \eta}{1+\alpha} \frac{B_{1 x}}{B_{\mathrm{o}}}+\frac{2 \alpha \xi}{1+\alpha} \frac{B_{1 y}}{B_{\mathrm{o}}}+\xi \varphi \\
\Omega u_{y}=\frac{\eta}{1+\alpha} \frac{P_{1}}{P_{\mathrm{o}}}-i \frac{2 \alpha \Theta}{1+\alpha} \frac{B_{1 z}}{B_{\mathrm{o}}}+\eta \varphi, \\
i \Omega u_{z}=\frac{1}{1+\alpha} \frac{d}{d \zeta} \frac{P_{1}}{P_{\mathrm{o}}}-\frac{2 \Theta}{1+\alpha} \frac{P_{1}}{P_{\mathrm{o}}}-i \frac{2 \alpha \eta}{1+\alpha} \frac{B_{1 z}}{B_{\mathrm{o}}}+\frac{2 \alpha}{1+\alpha} \frac{d}{d \zeta} \frac{B_{1 y}}{B_{\mathrm{o}}} \\
-\frac{4 \alpha \Theta}{1+\alpha} \frac{B_{1 y}}{B_{\mathrm{o}}}+\frac{d \varphi}{d \zeta}+2 \Theta \frac{\rho_{1}}{\rho_{\mathrm{o}}}, \\
\Omega \frac{B_{1 x}}{B_{\mathrm{o}}}=-\eta u_{x}, \\
\Omega \frac{B_{1 y}}{B_{\mathrm{o}}}=-i \frac{d u_{z}}{d \zeta}+i \Theta u_{z}+\xi u_{x}, \\
\Omega \frac{B_{1 z}}{B_{\mathrm{o}}}=-\eta u_{z},
\end{gathered}
$$




$$
\Omega \frac{P_{1}}{P_{\mathrm{o}}}=2 i \Theta u_{z}+\gamma\left(\xi u_{x}+\eta u_{y}\right)-i \gamma \frac{d u_{z}}{d \zeta}
$$

and finally

$$
\frac{d^{2} \varphi}{d \zeta^{2}}-\left(\xi^{2}+\eta^{2}\right) \varphi=2\left(1-\Theta^{2}\right) \frac{\rho_{1}}{\rho_{\mathrm{o}}} .
$$

Here we have taken $H, \sqrt{1+\alpha} c_{\mathrm{s}}(\equiv V)$ and $V / H$ as length, velocity and frequency units, respectively: $\xi=k_{x} H, \eta=k_{y} H, \zeta=z / H, u_{x}=v_{x} / V, u_{y}=v_{y} / V, u_{z}=v_{z} / V, \varphi=$ $\psi_{1} / V^{2}, \Omega=\omega(V / H)^{-1}$, and $\Theta \equiv \tanh \zeta$. Eliminating all the variables in favor of $u_{z}$ and $\varphi$, we combine equations (13) through (20) into the following two second-order ordinary differential equations:

$$
A_{2} \frac{d^{2}}{d \zeta^{2}} u_{z}+A_{1} \frac{d}{d \zeta} u_{z}+A_{0} u_{z}=B_{1} \frac{d}{d \zeta} \varphi+B_{0} \varphi
$$

and

$$
C_{2} \frac{d^{2}}{d \zeta^{2}} \varphi+C_{0} \varphi=D_{1} \frac{d}{d \zeta} u_{z}+D_{0} u_{z}
$$

Coefficients $A_{0}, A_{1}, A_{2}, B_{0}, B_{1}, C_{0}, C_{2}, D_{0}$, and $D_{1}$ are given in Appendix A.

\subsection{Boundary Conditions and Numerical Method}

To fix boundary conditions we will follow Goldreich \& Linden-Bell (1965) and Tomisaka \& Ikeuchi (1983). Let us suppose that the upper boundary of the disk is displaced from $z=$ $\pm z_{a}$ by a small amount $\delta z=s \exp \left[i\left(k_{x} x+k_{y} y-\omega t\right)\right]$ with $s \ll H$, which will then give us the vertical component of perturbation velocity at the boundary as

$$
v_{z}\left(z_{a}\right)=-i \omega \delta z
$$

An application of the Gauss flux theorem to the perturbed boundary gives us

$$
-k \psi_{1}\left(z_{a}\right)-\left.\frac{d \psi_{1}}{d z}\right|_{z_{a}-}=4 \pi G \rho_{\mathrm{o}}\left(z_{a}\right) \delta z
$$

since $\psi_{1}\left(z_{a}+\right)$ satisfies the Laplace equation in the rarefied halo.

A third condition comes from pressure continuity. It is the sum of the gas and the magnetic pressures that should be kept continuous at the boundary. By integrating $z$ component of the MHD momentum equation from $z_{a}$ to $z_{a}+\delta z$, one may have the total pressure at the perturbed boundary as

$$
P\left(z_{a}+\delta z\right)+\left.\frac{1}{8 \pi} B^{2}\right|_{z_{a}+\delta z}=P\left(z_{a}\right)+\left.\frac{1}{8 \pi} B^{2}\right|_{z_{a}}-\left.\rho_{\mathrm{o}}\left(z_{a}\right) \frac{d \psi_{\mathrm{o}}}{d z}\right|_{z_{a}} \delta z+O\left(\delta z^{2}\right) .
$$


Therefore, the total boundary pressure, $P_{\text {tot }}^{\text {disk }}$, calculated from the disk side becomes

$$
P_{\text {tot }}^{\text {disk }}\left(z_{a}+\delta z\right)=P_{\mathrm{o}}\left(z_{a}\right)+P_{1}\left(z_{a}\right)+\left.\frac{1}{8 \pi} B_{\mathrm{o}}^{2}\right|_{z_{a}}+\left.\frac{1}{4 \pi} B_{\mathrm{o}} B_{1 y}\right|_{z_{a}}-\left.\rho_{\mathrm{o}}\left(z_{a}\right) \frac{d \psi_{\mathrm{o}}}{d z}\right|_{z_{a}} \delta z
$$

to first order of $\delta z$. For the same boundary pressure, $P_{\text {tot }}^{\text {halo }}$, but calculated from the halo side, we may have

$$
P_{\text {tot }}^{\text {halo }}\left(z_{a}+\delta z\right)=P_{\mathrm{o}}\left(z_{a}\right)+\left.\frac{1}{8 \pi} B_{\mathrm{o}}^{2}\right|_{z_{a}}+\left.\frac{1}{4 \pi} B_{\mathrm{o}} B_{1 y}^{\text {halo }}\right|_{z_{a}}
$$

where perturbation of the halo gas pressure $P_{1}^{\text {halo }}\left(z_{a}\right)$ has been ignored, as the halo medium is thought to be very hot. Also ignored is the gravitational potential term, because the halo is filled with a rarefied medium. By equating $P_{\text {tot }}^{\text {disk }}$ to $P_{\text {tot }}^{\text {halo }}$ at the boundary, we finally have the pressure continuity condition as

$$
P_{1}\left(z_{a}\right)+\left.\frac{1}{4 \pi} B_{\mathrm{o}} B_{1 y}\right|_{z_{a}}-\left.\rho_{\mathrm{o}}\left(z_{a}\right) \frac{d \psi_{\mathrm{o}}}{d z}\right|_{z_{a}} \delta z=\left.\frac{1}{4 \pi} B_{\mathrm{o}} B_{1 y}^{\text {halo }}\right|_{z_{a}} .
$$

Now $B_{1 y}^{\text {halo }}$ has to be specified. From the induction equation [Eq. (10)] we obtain

$$
\vec{B}_{1}=-\frac{k_{y}}{\omega} v_{x} B_{\mathrm{o}} \hat{e}_{x}-\left[\frac{i}{\omega} \frac{\partial}{\partial z}\left(v_{z} B_{\mathrm{o}}\right)-\frac{k_{x}}{\omega} v_{x} B_{0}\right] \hat{e}_{y}-\frac{k_{y}}{\omega} v_{z} B_{\mathrm{o}} \hat{e}_{z} .
$$

On the other hand, no current density is expected in the halo, because matter density there is extremely low. Therefore, $\vec{B}_{1}^{\text {halo }}$ can be given by magnetic potential $\phi_{m}$ simply as

$$
\vec{B}_{1}^{\text {halo }}=B_{\mathrm{o}} \nabla \phi_{m}
$$

with

$$
\phi_{m}=\mathrm{A} \exp \left(i k_{x} x+i k_{y} y-k z\right) \text { and } k^{2}=k_{x}^{2}+k_{y}^{2} .
$$

Equation (28) tells us that the vertical component of the magnetic field becomes $B_{1 z}^{\text {disk }}=$ $-\left(k_{y} / \omega\right) v_{z} B_{\mathrm{o}}$ at the disk side boundary. From the halo side, Equation (29) shows $B_{1 z}^{\text {halo }}=$ $-k B_{\mathrm{o}} \phi_{m}$. By equating $B_{1 z}^{\text {disk }}$ to $B_{1 z}^{\text {halo }}$, we have $\phi_{m}=\left(k_{y} / k \omega\right) v_{z}$ and the azimuthal field component $B_{1 y}^{\text {halo }}=i\left(k_{y}^{2} / k \omega\right) v_{z} B_{\mathrm{o}}$. And its substitution for $B_{1 y}^{\text {halo }}$ in equation (27) finally gives the pressure continuity condition as:

$$
P_{1}\left(z_{a}\right)+\left.\frac{1}{4 \pi} B_{\mathrm{o}} B_{1 y}\right|_{z_{a}}-\left.\rho_{0}\left(z_{a}\right) \frac{d \psi_{\mathrm{o}}}{d z}\right|_{z_{a}} \delta z=\left.i \frac{B_{\mathrm{o}}^{2}}{4 \pi}\right|_{z_{a}} \frac{k_{y}^{2}}{k \omega} v_{z}\left(z_{a}\right) .
$$

In terms of the dimensionless variables the upper boundary conditions [Eqs. (23), (24), and (31)] take the following forms:

$$
-i \Omega \delta \zeta=u_{z}\left(\zeta_{a}\right),
$$




$$
-k \varphi\left(\zeta_{a}\right)-\left.\frac{d \varphi}{d \zeta}\right|_{\zeta_{a}}=2 \operatorname{sech}^{2}\left(\zeta_{a}\right) \delta \zeta
$$

and

$$
\begin{gathered}
\left.\quad \frac{1}{2} \gamma i \Omega \mathcal{D} \cosh ^{2} \zeta_{a} \frac{d^{2} \varphi}{d \zeta^{2}}\right|_{\zeta_{a}}-i \Omega\left(\frac{1}{2} \gamma \mathcal{D} k^{2} \cosh ^{2} \zeta_{a}-2 \alpha \xi^{2} \Omega^{2}\right) \varphi\left(\zeta_{a}\right) \\
-2(1+\alpha) \Theta i \Omega \mathcal{D} \delta \zeta+\left.2 \alpha\left(\Omega^{2}-\frac{2 \alpha}{1+\alpha} \eta^{2}\right)\left(\Omega^{2}-\frac{\gamma}{1+\alpha} \eta^{2}\right) \frac{d u_{z}}{d \zeta}\right|_{\zeta_{a}} \\
+\left[2 \Theta(\gamma-1) \mathcal{D}+\frac{2 \alpha \eta^{2}}{k} \mathcal{D}-2 \Theta \alpha\left\{\Omega^{4}-\left(\frac{\gamma-2}{1+\alpha} \xi^{2}+\frac{2 \alpha+\gamma}{1+\alpha} \eta^{2}\right) \Omega^{2}+\frac{2 \alpha \gamma}{(1+\alpha)^{2}} \eta^{2} k^{2}\right\}\right] u_{z}\left(\zeta_{a}\right)=0
\end{gathered}
$$

where

$$
z_{a} / H=\zeta_{a} \text { and } \mathcal{D}=\Omega^{4}-\frac{2 \alpha+\gamma}{1+\alpha} k^{2} \Omega^{2}+\frac{2 \alpha \gamma}{(1+\alpha)^{2}} \eta^{2} k^{2}
$$

At the midplane, parity conditions should be specified: If symmetric (odd-parity) solution is sought for vertical velocity $u_{z}$, it should satisfy

$$
\left.\frac{d \varphi}{d \zeta}\right|_{\zeta=0}=0 \text { and } u_{z}(\zeta=0)=0
$$

at the midplane; while anti-symmetric (even-parity) one requires

$$
\varphi(\zeta=0)=0 \text { and }\left.\frac{d u_{z}}{d \zeta}\right|_{\zeta=0}=0 .
$$

The odd-parity solutions are mirror symmetric with respect to the midplane, and the evenparity ones are of the midplane crossing type.

Along with the upper-boundary continuity and the midplane parity conditions, equations (21) and (22) comprise an eigenvalue problem for $\Omega^{2}$. For given perturbation wave vector $(\xi, \eta)$ we take a trial value for $\Omega^{2}$, and integrate the equations from $\zeta=\zeta_{a}$ to $\zeta=$ 0 with the fourth-order Runge-Kutta method. If the resulting solution satisfies the chosen parity condition at $z=0$, the trial is adopted as the eigenvalue. If not, we take another trial and perform the same integration until the solution fulfills the midplane parity condition.

The resulting growth rate squared, $-\Omega^{2}$, is illustrated as a function of $\nu_{x}=\xi \tanh \zeta_{a}$ and $\nu_{y}=\eta \tanh \zeta_{a}$ (not of $\xi$ and $\eta$ ), which will keep the slope of dispersion relation the same in limits $\nu_{x} \rightarrow 0$ or $\nu_{y} \rightarrow 0$, regardless of the disk thickness (Elmegreen \& Elmegreen 1978). If we notice total column density is $2 \rho_{\mathrm{o}}(0) \times H$ for unbound isothermal disk and $2 \rho_{\mathrm{o}}(0) \times H \tanh \zeta_{a}$ for halo-bound one, we may call $\nu_{x}$ and $\nu_{y}$ normalized wave numbers that are effective for the halo-bound disk. The three parameters $\left(\alpha, \gamma, \zeta_{a}\right)$ characterize the disk system and the normalized effective wave numbers $\left(\nu_{x}, \nu_{y}\right)$ do the perturbation. 


\section{CONVECTION, PARKER AND JEANS INSTABILITIES}

Whenever a need arises to differentiate the undular-mode instability driven by external gravity from that by self gravity, we will use the term classical Parker, for which both oddand even-parities are applicable. In the context of self gravity, we will call the instability triggered by the same undular mode but even-parity perturbations as simply the Parker or the pure Parker instability, while reserving the term Parker-Jeans for the odd-parity undular mode. On the other hand, the instability triggered by short wavelength perturbations of the interchange mode will be called broadly as convection. Long wavelength perturbations of the same mode may of course activate the gravitational instability. In this section we will examine how the convection, the pure Parker instability, and the Jeans gravitational instability compete or collaborate with each other in forming or de-structuring large-scale structures.

\subsection{Undular Mode}

To have a comparison standard we first calculate the dispersion relation for the pure gravitational instability by taking $\alpha=0, \nu_{x}=0$ and imposing the odd-parity condition. For several cases of disk thickness, Figure 1a illustrates how the normalized growth rate of the gravitational instability changes with the azimuthal effective wave number. In general, the Jeans instability grows faster in thicker disk. However, once it is thicker than about 4 times the scale height, the growth rate becomes almost independent of the disk thickness.

With the same set of system parameters except for $\alpha=1.0$, we calculate $-\Omega^{2}$ again

as a function of $\nu_{y}$. The resulting dispersion relations for odd- and even-parity solutions are shown, in Figure 1b, by solid and dotted lines, respectively. Because even-parity cannot activate the gravitational instability (Simon 1965; Elmegreen \& Elmegreen 1978), by simply changing the parity condition from even to odd, we may easily differentiate role of the Parker instability from that of the Jeans in the dispersion relation. The dotted lines in Figure $1 \mathrm{~b}$ are, therefore, for the Parker instability only; while the solid ones are for the case where both the Parker and the Jeans instabilities operate simultaneously. This led us to coin the term Parker-Jeans especially for the odd-parity undular mode.

A comparison of the solid lines in Figures $1 \mathrm{a}$ and $1 \mathrm{~b}$ indicates that the presence of magnetic fields in moderately thick disks boosts the growth rate of the gravitational instability significantly. The Parker and the Jeans instabilities interact constructively in the disks thicker than, say, one half the scale height; while in the disks thinner than that, the same magnetic fields now add rigidity or extra pressure to the gas, thereby hindering growth of 
the gravitational instability. With marginal stability analysis (see Appendix C) we have determined the critical thickness, over which the two instabilities interact constructively or destructively: It is $0.549 H$ for a moderately magnetized $(\alpha \simeq 1)$, isothermal $(\gamma=1)$ disk. As can be seen from the dotted lines in Figure 1b, growth rate of the Parker instability depends on thickness more sensitively than that of the Jeans instability. The dotted line almost coinciding with the horizontal axis is for $\zeta_{a}=1$, and the dispersion relation of evenparity becomes hardly noticeable for $\zeta_{a} \leq 1.0$. Comparison of the dotted (even-parity) and solid (odd-parity) lines in Figure 1b suggests that a marked increase can be achieved in the growth rate of the Parker instability by triggering the gravitational instability. This opens up a possibility of alleviating the time scale problem of the classical Parker instability.

To understand how the magnetic field strength modifies the dispersion relation for the undular mode, we fix the disk thickness at $5 H$ and vary $\alpha$ over $0,1,2,3$, and 4 . The resulting $-\Omega^{2}$ is plotted, in Figure 2, against the effective azimuthal wave number. As before the solid and dotted lines are for odd- and even-parity solutions, respectively. It is clear from the figure that the maximum growth rate of the Parker instability increases with field strength (Notice difference in the ordinates between Figures 1 and 2.). Under an influence of vertical acceleration, the undulated field lines give the magnetic buoyancy a chance to unload the gas material off their shoulders towards the midplane valleys. As the field strength increases, so does the buoyancy, and hence the system becomes highly unstable to the perturbations of undular mode. The resulting escalation of the Parker instability then boosts development of the gravitational instability. The maximum growth rate of the oddparity solutions, therefore, increases significantly with $\alpha$. The critical wave number for marginal stability is known to increase with $\alpha$ as $(1+2 \alpha)^{1 / 2}$ (Stodólkiewicz 1963), which also indicates the constructive nature of Parker and Jeans interaction.

We now turn our attention to the remaining system parameter, $\gamma$, which was introduced as a measure of material rigidity. To trigger the classical Parker instability under external gravity, effective ratio of the specific heats for the perturbed material should be less than a certain critical value, $\gamma_{\mathrm{c}}$. Many authors have shown $\gamma_{\mathrm{c}}=1+\alpha$ under external gravity (Newcomb 1961; Parker 1966; Gilman 1970; Kim \& Hong 1998). In a recent study Lee (2002) proved that the critical value takes the same $1+\alpha$ under the influence of self gravity as well. To somewhat dramatize the effect of $\gamma$ on the growth rate we decided to fix $\alpha$ at a rather small value 0.1 and examined the dispersion relation with $\gamma$ varying 0.68 through 1.1. The solid and dotted lines in Figure 3 are for the odd- and even-parity solutions, respectively. For soft materials the dispersion curve exhibits two maxima: The gravitational instability is responsible for the peak occurring at smaller wave number and the Parker is for the other at larger one. As $\gamma$ increases, height of the Parker peak decreases. This is because, as the material becomes harder, the magnetized gas system becomes more stable to 
the undular mode perturbation. If $\gamma$ exceeds its critical value, the Parker instability cannot be triggered at all. Owing to a heightening activity of the Parker instability in softer media, the Jeans peak becomes taller as $\gamma$ decreases.

For most cases shown in Figure 3, the dotted lines of even-parity match smoothly with the solid lines of odd-parity. For somewhat large $\gamma$ 's, however, the even-parity perturbations of undular mode grow a little faster than the odd-parity ones. Under self gravity the growth rate difference between the even- and odd-parity solutions is too small to render any significant consequences to the spatial distribution of the resulting condensations with respect to the midplane. In the case of classical Parker instability, however, the even-parity perturbations grow substantially faster than the odd-parity ones. Therefore, the condensations, if they were generated by the classical Parker instability, would place their maximum density points on the northern and southern sides of the midplane alternatively (Kim et al. 2000).

Figure 3 suggests that the undular mode of odd-parity triggers both the Jeans and the Parker instabilities in the $\gamma=0.68$ disk, for example. The two most rapidly growing perturbations in the disk have $\lambda_{y} \simeq 8.6 H$ for the Jeans instability and $\lambda_{y} \simeq 3.5 H$ for the Parker case. On the other hand, the perturbations of the same undular mode with the same $\lambda_{y} \simeq 8.6 \mathrm{H}$ but of even-parity would turn off the gravitational instability and drive the system to develop only the Parker. We have shown, in Figures $4 \mathrm{a}$, $4 \mathrm{~b}$ and $4 \mathrm{c}$, eigen-solutions for the cases with $\lambda_{y} \simeq 8.6 H$ of odd-parity, with $\lambda_{y} \simeq 3.5 H$ of odd-parity, and with $\lambda_{y} \simeq$ $8.6 H$ of even-parity, respectively. In the left panel, the color changes from dark red to white as density increases, and the numbers marked on each contours represent logarithm of the density perturbation, $\rho_{1}(y, z)$, measured in units of the initial equilibrium density, $\rho_{\mathrm{o}}(z=0)$, at the midplane. In the right hand panels, contours in thin solid lines are for the magnetic fields and the ones in thick lines delineate the halo boundary. Small arrows inside the panels represent velocity vectors, a big arrow on top of frame $a$ and $b$ corresponds to two times the sound speed, and that of frame $c$ does just one.

An action of the self gravity is apparent from the velocity pattern in Figure 4a. In the region of inflated field lines the magnetic buoyancy drives matter to rise against the self gravity; while in the magnetic valley the self gravity does the driving in an opposite direction and collects material towards the center. We see motions taking place everywhere in the $y z$-plane. Even in the midplane one may witness a converging motion, which is made possible by the self gravity and cannot be expected under the external gravity. As a final product of the Jeans instability the system will bear out a dense clump centered at $\mathrm{Y} \simeq 4.3$. Velocity vectors in Figure 4b exhibit a pattern markedly different from the ones in Figure 4a. Magnitude of the velocity vectors quickly diminishes towards central plane, and they hardly exhibit any motions in the midplane. The magnetic field lines in low altitudes maintain 
their initial configuration almost intact. Features of the perturbed density, the magnetic fields and the velocity patterns in Figure $4 \mathrm{~b}$ are all very similar to the corresponding cases of the classical Parker instability that is driven by an external gravity of linear model (Lee 1993). As a final product of the Parker instability the system will bear out clumps centered at $\mathrm{Y} \simeq 1.7,5.2$ and 8.5. The iso-density contours for odd-parity solutions in Figures $4 \mathrm{a}$ and $4 \mathrm{~b}$ demonstrate dense clumps formed right in the midplane. The deformation of boundary is closely related to the enhanced density near the midplane (Elmegreen \& Elmegreen 1978). A rise and a fall of the boundary surface from its initial equilibrium height $z_{a}=5 \mathrm{H}$ accompany a fall and a rise in the midplane density, respectively.

Eigen-solutions of the even-parity shown in Figure 4c make a marked contrast to the odd-parity solutions in Figures $4 \mathrm{a}$ and $4 \mathrm{~b}$. The upper and lower boundaries make the riseand-fall motions in unison. Active motions are apparent only in high above the midplane. These features are very much reminiscent of the Parker instability driven by a linear model of external gravity (Lee 1993). It is quite clear that the classical Parker instability may not be the formation mechanism of such large-scale structures as GMCs or HI superclouds. The GMCs are attributes of the Galactic midplane, while the Parker instability generates actions at high altitudes. To have such dense structures as GMCs in the disk central plane, we need to invoke the self gravity not only to drive the Parker undular mode but also to trigger the Jeans gravitational instability.

As far as forming large-scale structures is concerned, the odd-parity undular mode deserves our special attention, because it will drive the system to develop the Jeans gravitational instability and the Parker instability simultaneously. If the two instabilities work constructively, they would generate dense condensations in the central plane. On the other hand, the undular mode perturbations of even-parity drive the system to develop only the Parker instability, whose activities are confined mostly to high altitude regions, where GMCs ar not generally found. The question then is whether the resulting condensations of the odd-parity solutions are safe from disruption by convective motions.

\subsection{Interchange Mode}

To construct dispersion relation for the pure interchange mode, we fix azimuthal wave

number at zero $\left(\nu_{y}=0\right)$ and calculate the growth rate as a function of effective radial wave number $\nu_{x}$. The results for the odd-parity solutions are shown, in Figure 5, for the disks of a moderately magnetized ( $\alpha=1$ ), self-gravitating, isothermal gas medium. As was seen from Figure 1a for the non-magnetized gas disk, the maximum growth rate generally increases with disk thickness. However, for the disks thicker than $\sim 5 H$, the dispersion relations for 
different thickness are hardly distinguishable from each other. Once the upper boundary is placed beyond a certain distance from the midplane, development of the gravitational instability in the densest part of the system doesn't depend much on where the boundary is located. In disks thinner than $\sim H / 3$, the growth rate again becomes independent of the thickness. In such thin halo-bound disks, there is practically no gradient in density over vertical distance, and the gas acts like an incompressible fluid (Goldreich \& LyndenBell 1965; Elmegreen \& Elmegreen 1978; Lubow \& Pringle 1993; Nagai et al. 1998; Lee 2002). A sizeable difference exists in the maximum growth rate between non-magnetized (see Figure 1a) and magnetized (see Figure 5) disks. Perturbations of the interchange mode make density and magnetic flux change in phase, and thus activate fast magneto-acoustic waves to propagate in the radial direction. Since the fast waves tend to disperse enhanced density, the gravitational instability triggered by the interchange mode ought to grow slower than that by the undular mode. This is the reason for the difference between the two modes.

We saw from Figure 2 that the existence of magnetic fields boosts the gravitational instability triggered by the undular mode. To check whether the same boosting is possible for the interchange mode, we calculate $-\Omega^{2}$ of the odd-parity solutions for $\alpha=0,1,2$, and 3. As shown in Figure 6, the maximum growth rate decreases with $\alpha$. This trend is opposite to the case of the undular mode. With the interchange perturbations the magnetic fields have no means for adding extra material to the densest region of the disk; instead they act to disperse the enhanced density by driving the fast magneto-acoustic waves. Therefore, the growth rate of the interchange mode decreases with increasing field strength.

In all the cases illustrated in Figures 5 and 6, the magnetic field is too strong to satisfy the criterion, $\gamma<1-\alpha$, for convection. Originally this criterion is known to hold for disks under external gravity (Newcomb 1961; Parker 1967; Zweibel \& Kulsrud 1975; Asséo et al. 1978; Lachièze-Rey et al. 1978). As for the $\gamma_{\mathrm{c}}$ criterion for the classical Parker instability, Lee (2002) showed that the same convection criterion, $\gamma<1-\alpha$, is applicable to the self gravity case.

Adopting a weakly magnetized $(\alpha=0.1)$ disk to see the convective motions and assigning small values to the ratio of effective specific heats to meet the convection criterion, we calculated the dispersion relation as a function of $\nu_{x}$. The results are shown in Figure 7 , where the solid and the dotted lines are for the odd- and the even-parities, respectively, and on each curve $\gamma$ is marked. The well-defined peak corresponds to the Jeans instability; while the monotonically increasing part is owing to the convective instability. The evenparity perturbations cannot trigger the gravitational instability but certainly can activate the convection. After passing a minimum the growth rate first increases monotonically with radial wave number and reaches a finite value asymptotically as the wave number further 
increases: Arrows outside the frame indicate the limiting values of the growth rate. Under external gravities the interchange mode perturbations of the shortest wavelengths are to grow most rapidly and deprive the undular mode of the chance to form large-scale structures. Under the self gravity, however, the maximum growth rate of the gravitational instability can be higher than the limiting growth rate of the convective instability. The self gravity may thus have a chance fully to suppress convective motions. This is a most important attribute of the self-gravitating disk. Since the external gravity exerts acceleration of fixed strength only in the vertical direction, there is no means of de-structuring the vertical sheets generated by the convective motions. Once a substantial degree of density enhancement is achieved, however, the ever increasing self gravity can provide the system with a means for redirecting the vertical motions towards the dense mass center.

Even if the convection can grow faster than the Jeans instability in linear stage of the disk evolution, particularly in 'soft' medium of small $\gamma$ 's, the convective motion won't prevent the system from forming dense structures in the midplane for two reasons: First, no buoyancy force is available in the midplane and hence no convective motions can take place there. Second, the acceleration due to self gravity increases with density enhancement. Therefore, once the gravitational instability is triggered by odd-parity perturbations, it will eventually dominate the disk over the convection.

The eigen-solution of the odd-parity interchange mode was constructed for the case with $\gamma=0.68$ and $\nu_{x}=0.6\left(\lambda_{x}=10.5 \mathrm{H}\right)$, which corresponds the well-defined growth rate peak in Figure 7. The same was done of the even-parity interchange mode with $\nu_{x}=3.0\left(\lambda_{x}\right.$ $=2.1 \mathrm{H}$ ), which is a convection dominating case. Figures $8 \mathrm{a}$ and $8 \mathrm{~b}$ compare the two sets of eigen-solutions in the $x z$-plane. The left and right panels illustrate density and velocity fields, respectively, and the color changes from dark red to white as density increases. The numbers marked to thin solid lines on the left are logarithms of $\rho_{1}(x, z) / \rho_{\mathrm{o}}(z=0)$. The thick solid lines on the right demarcate the disk-halo boundary, the arrows inside the frames represent velocity vectors, and the horizontal arrow on top of each frame is a measure of the sound speed.

A well-defined density clump in Figure 8a is obviously a result of the converging motions driven by the Jeans instability. The velocity pattern in Figure 8a closely resembles the same pattern in Figure 4a: Converging motions are evident all over the $x z$-plane in Figure 8a and the $y z$-plane in Figure 4a. Figure 8a suggests that once the interchange mode perturbation generates a slight density enhancement at a midplane point, towards which the self gravity would collect material and form a density clump. On the other hand, we don't expect from Figure 8b such clump to form in the midplane: Motions are absent from the low altitude region. 


\subsection{Mixed Mode}

We now calculate the growth rate as a function of $\nu_{x}$ and $\nu_{y}$. The results are presented in a three dimensional surface plot of $-\Omega^{2}$ over the wave number domain. As our interest is primarily in forming large-scale structures in the Galactic ISM disk, we fix the disk thickness at $\zeta_{a}=5$, which is sufficiently larger than the critical value, 0.549 (see §3.1). Figures 9a and $9 \mathrm{~b}$ are for even-parity solutions with $(\alpha=1, \gamma=1)$ and $(\alpha=0.1, \gamma=0.8)$, respectively. The surface plot in Figure 9a looks like a long 'tunnel', and we already met, in Figure $1 \mathrm{~b}$, the dispersion curve outlining the tunnel 'entrance' at $\nu_{x}=0$. Cross-section of the tunnel cut perpendicularly to the $\nu_{x}$-axis describes the dispersion relation of the Parker instability triggered by undular portion of the mixed mode perturbation. Please note that the tunnel ridge inclines slowly with $\nu_{x}$. This is owing to the interchange part of the mixed mode. The pure interchange mode (i.e., $\nu_{y}=0$ ) doesn't trigger the magnetic RayleighTaylor instability (cf., Chandrasekhar [1961] for this terminology), since this disk does not satisfy the $\gamma<1-\alpha$ criterion for this particular mode. But interchange part of the mixed perturbation does trigger the convection everywhere in the $\left(\nu_{x}, \nu_{y}\right)$ plane except at $\nu_{y}=0$, since another condition $\gamma<1+\alpha$ is required for truly mixed mode. It is the convection that makes the 'ridge' incline with increasing $\nu_{x}$. In the case shown in Figure 9b, the magnetic Rayleigh-Taylor instability is activated by the pure interchange mode, as the disk satisfies now the condition $\gamma<1-\alpha$. This is why the tunnel wall of Figure 9b is partially 'open' on the $\nu_{y}=0$ side.

The three-dimensional plots shown in Figures $9 \mathrm{c}$ and $9 \mathrm{~d}$ are for the odd-parity solutions but with the same set of system parameters as in Figures 9a and 9b, respectively. We may relate the two peaks located on the $\nu_{x^{-}}$and $\nu_{y^{-}}$axes to the Jeans and the Parker-Jeans instabilities, respectively. The peak with $\nu_{x}=0$ is significantly higher than the one with $\nu_{y}=0$, since the undular mode perturbation provides the Parker instability with favorable field configurations to assist the Jeans. On the other hand, the pure interchange mode may not get any boosting from the Parker instability. Consequently, the pure Jeans peak on the $\nu_{x}$-axis is always lower than the Parker-Jeans on the $\nu_{y}$-axis. The long ridge along $\nu_{y} \simeq 1$ is of course owing to the convection-assisted Parker instability; as for the case of even-parity solutions, the convection makes the ridge height incline with $\nu_{x}$. But the ridge reaches a finite level in the limit $\nu_{x} \rightarrow \infty$.

When mixed mode perturbations of the odd-parity are given to the self-gravitating, magnetized, gaseous disk, the Jeans, Parker and convective instabilities may all develop in the disk, and their relative importance depends on the $\left(\nu_{x}, \nu_{y}\right)$ combination. We have constructed, from Figure 9c, a plot of equal growth-rate contours in the $\left(\nu_{x}, \nu_{y}\right)$ plane and presented the resulting contour map in Figure 10. The thick dashed-line is for a special 
contour, whose growth rate is equal to the the highest ridge level: Any perturbations with wave numbers falling within the dashed-line boundary would grow faster than the ones outside. Therefore, with such perturbations, a collaboration of the Parker and the Jeans instabilities may well suppress the convection and the disk will bear out large-scale structures.

In a recent two-dimensional MHD simulation, Kim \& Ostriker (2002) followed nonlinear responses of a self-gravitating, magnetized, differentially rotating, thin disk to an externally imposed spiral potential, and demonstrated regularly spaced spurs jotting out initially in an almost perpendicular direction to the arm. Although nonlinear interaction of the ParkerJeans instability and the spiral shock wave is out of the scope of this paper, it should be pointed out that the highest point of the growth rate surface is located on the $\nu_{y}$-axis (see Figure 10). This is always so, if the disk is thicker than $\sim 0.549 H$ (see Appendix C). The resulting structure is, therefore, of a cylindrical shape with its long axis being aligned perpendicularly to the direction of magnetic field lines or spiral arm (Nagai et al. 1998; Lee \& Hong 1999; Chou et al. 2000). It is then suggestive that the cylindrical structures of this study will make the spurs in the context of differential rotation and density wave. The odd-parity undular mode seems to have tailored the regular spacing and initial alignment of the spurs.

\section{FORMATION OF LARGE ISM STRUCTURES}

A replacement of driving agent of the Parker instability from the external to the self gravity opens up a new route, through which the instability may work constructively with the Jeans gravitational instability to form large-scale structures in the Galactic ISM disk. In a qualitative sense, the undular mode odd-parity solutions seem to have all the means of alleviating the difficulties involved in the time scale, length scale and density enhancement of the classical Parker instability. In this section we will extend our qualitative understandings of the dispersion relation to making quantitative estimates of the various scales.

In this study the Galactic ISM disk is characterized by midplane mean density $n_{\mathrm{o}}$ of hydrogen nuclei, $r m s$ dispersion $c_{\mathrm{s}}$ of "cloudlet" velocities, ratio $\alpha$ of magnetic to gas pressure, and thickness $z_{\mathrm{a}}\left(=\zeta_{\mathrm{a}} H\right)$ of the disk. Unless $\tanh \zeta_{\mathrm{a}}$ is substantially less than unity, details of the dynamics involved with the Parker and the Jeans instabilities do not depend on the thickness, which leaves $n_{\mathrm{o}}, c_{\mathrm{s}}$ and $\alpha$ as the system parameters of principal importance. We will examine how these three parameters control the minimum growth time of the ParkerJeans instability, its corresponding length scale, and the mass associated with the length. 


\subsection{A Comparison Standard of the ISM Parameters}

To calculate dynamically important scales of the Parker-Jeans instability, we need to establish a 'standard' set for the system parameters. By taking averages of various observations, Ferrière $(1995,1998 \mathrm{a}, 1998 \mathrm{~b})$ presented space-averages of the ISM parameters, which may serve us a starting point for setting up the standard. According to his 1998 results, the midplane equilibrium number density of hydrogens in the solar neighborhood is $n_{\mathrm{o}}=$ $1.17 \mathrm{~cm}^{-3}$, which includes all forms of hydrogens residing in the four ISM phases and the molecular medium as well. With an inclusion of helium ( $9 \%$ of hydrogen by number), the mean molecular weight of ISM per hydrogen nucleus becomes $\mu_{\mathrm{ISM}}=1.36$, which makes $n_{\mathrm{o}} \mu_{\mathrm{ISM}}=1.59 \mathrm{~cm}^{-3}$. As a comparison standard for density we will simply take $n_{\mathrm{o}} \mu_{\mathrm{ISM}} \simeq$ $2 \mathrm{~cm}^{-3}$ (cf., Holmberg \& Flynn 2000; Kulkarni \& Heiles 1987).

Excluding the molecular medium, Ferrière (1998a) determined the total thermal pressure of the four ISM phase materials to be $4.81 \times 10^{-13} \mathrm{ergs} \mathrm{cm}^{-3}$. As the one-dimensional turbulent velocity he took $4.5 \mathrm{~km} \mathrm{~s}^{-1}$ and $6.9 \mathrm{~km} \mathrm{~s}^{-1}$ for the molecular and the cold neutral media, respectively, and estimated the total interstellar turbulent pressure to be $6.31 \times 10^{-13} \mathrm{ergs} \mathrm{cm}^{-3}$. The sum of thermal and turbulent pressures becomes then $11.12 \times 10^{-13} \mathrm{ergs} \mathrm{cm}^{-3}$. If his estimate of the cosmic ray pressure $9.6 \times 10^{-13} \mathrm{erg} \mathrm{cm}^{-3}$ is included in the pressure count, the total amounts to $20.7 \times 10^{-13} \mathrm{ergs} \mathrm{cm}^{-3}$. With the standard density $n_{\mathrm{o}} \mu_{\mathrm{ISM}} \simeq 2 \mathrm{~cm}^{-3}$, this formally requires the isothermal sound speed or the velocity dispersion to be $7.9 \mathrm{~km} \mathrm{~s}^{-1}$. This seems to be an over-estimate of reality, particularly for ISM near the central plane. Owing to the neglect of cosmic rays in this study, we will include only the thermal and the turbulent pressures in the isothermal equation of state and take $c_{\mathrm{s}} \simeq 5.8 \mathrm{~km} \mathrm{~s}^{-1}$ as a better choice for the comparison standard.

For the magnetic pressure Ferrière (1998a, 1998b) gave $10.3 \times 10^{-13} \mathrm{ergs} \mathrm{cm}^{-3}$. In terms of magnetic field strength, this is equivalent to $5.1 \mu \mathrm{G}$, which is significantly stronger than frequently quoted $3 \mu \mathrm{G}$ (cf., Spitzer 1978). If we accept all the pressure values of his estimate, $\alpha$ becomes 0.50; if the cosmic ray pressure is not counted, the ratio becomes 0.93 . On the other hand, if we take $3 \mu \mathrm{G}$ for the field strength and count only the thermal and the turbulent pressures, then the magnetic-to-gas pressure ratio becomes 0.3. Because many uncertainties are involved in deducing the cosmic ray and the magnetic pressures from the observed distribution of synchrotron emissivity (Ferrière 1995, 1998a) and because a proper treatment of cosmic ray behaviors is beyond the scope of current study (cf., Ryu et al. 2003), $\alpha$ may not be fixed at a specific value at this stage. Instead we will examine how the scales vary with $\alpha$, and chose $\left(n_{\mathrm{o}} \mu_{\mathrm{ISM}} \simeq 2 \mathrm{~cm}^{-3}, c_{\mathrm{s}} \simeq 5.8 \mathrm{~km} \mathrm{~s}^{-1}, 0 \leq \alpha \leq 1\right)$ as the comparison standard. 


\subsection{Time, Length and Mass Scales}

A simple comparison of the odd-parity undular mode solutions to the even-parity ones will tell us how much change the constructive interplay between Parker and Jeans instabilities can bring to the growth time and the length scale. We have listed in Table 1 the normalized maximum growth rates $\left(\Omega_{\max }^{\mathrm{ODD}}, \Omega_{\max }^{\mathrm{EVN}}\right)$ and their corresponding wave numbers $\left(\nu_{y, \max }^{\mathrm{ODD}}, \nu_{y, \max }^{\mathrm{EVN}}\right)$ for a selected set of $\alpha$ values. Superscripts "ODD" and "EVN" can be identified with the Parker-Jeans and the Parker instabilities, respectively. The table was prepared with $\gamma=1$.

The ratio given in the fourth column demonstrates that a sizeable reduction is made in the growth time by the self gravity. In weakly magnetized disks the time scale is reduced to one third the case of pure Parker instability. Since long wavelength perturbations are prone to trigger the gravitational instability, we expect the length scale of the Parker-Jeans to be longer than that of the Parker instability. Indeed the ratio, $\lambda_{y, \max }^{\mathrm{ODD}} / \lambda_{y, \max }^{\mathrm{EVN}}$, in the last column shows that an about $30 \%$ increase in the length scale can be achieved by the Parker-Jeans instability. The relative increase in the length scale varies little with $\alpha$; while the enhancement in the growth rate changes significantly with the magnetic field strength.

The minimum growth time, $\tau_{\mathrm{GRW}}=\left(H / c_{\mathrm{s}}\right)\left|\Omega_{\max }\right|^{-1}$, is given by

$$
\tau_{\mathrm{GRW}} \simeq 3.8 \times 10^{7} \text { years }\left[\frac{1+\alpha}{n_{\mathrm{o}} \mu_{\mathrm{ISM}} \mathrm{cm}^{3}}\right]^{1 / 2} \frac{1}{\left|\Omega_{\max }(\alpha)\right|} .
$$

Because the disk scale height is directly proportional to $c_{\mathrm{s}}$, the time scale doesn't depend on it explicitly. Substituting the $\alpha$-dependent $\Omega_{y, \max }^{\text {ODD }}$ in Table 1 for the maximum growth rate, we examined how the growth time would change with $\alpha$. Results are shown by thick solid line of Figure 11. The two lower curves in thin solid lines are to be identified with specific cases of $n_{\mathrm{o}} \mu_{\mathrm{ISM}}=2 \mathrm{~cm}^{-3}$ and $3 \mathrm{~cm}^{-3}$. Please note that the system parameter $n_{\mathrm{o}} \mu_{\mathrm{ISM}}$ is absorbed in the ordinate for the thick solid line. To read the growth time scales for the latter two cases directly off the thin lines, the $n_{\mathrm{o}} \mu_{\mathrm{ISM}}$ factor should be ignored.

In terms of the system parameters the scale height $H$ becomes

$$
H \simeq 1.9 \times 10^{2} \mathrm{pc}\left[\frac{1+\alpha}{n_{\mathrm{o}} \mu_{\mathrm{ISM}} \mathrm{cm}^{3}}\right]^{1 / 2}\left[\frac{c_{\mathrm{s}}}{5 \mathrm{~km} \mathrm{~s}^{-1}}\right],
$$

which serves as the fundamental length for our disk system. The perturbation wavelength, $\Lambda=2 \pi H \nu_{\max }^{-1}$, corresponding to the maximum growth rate is given by

$$
\Lambda \simeq 1.2 \mathrm{kpc}\left[\frac{1+\alpha}{n_{\mathrm{o}} \mu_{\mathrm{ISM}} \mathrm{cm}^{3}}\right]^{1 / 2}\left[\frac{c_{\mathrm{s}}}{5 \mathrm{~km} \mathrm{~s}^{-1}}\right] \frac{1}{\nu_{y, \max }(\alpha)} .
$$

The observed mean separation of the large-scale structures can be compared directly with $\Lambda$, if $\nu_{y, \max }$ is associated with the odd-parity solution. In the case of even-parity, however, 
the observations should be compared with $\Lambda / 2$. When $\nu_{y \text {, max }}^{\text {ODD }}$ in Table 1 is substituted for $\nu_{y, \max }(\alpha)$ in the above equation, the length scale varies with $\alpha$ as in Figure 12 , where $n_{\mathrm{o}} \mu_{\mathrm{ISM}}$ and $c_{\mathrm{s}}$ are absorbed in the calculation of ordinates for the thick solid line. To read the length scales for the two lower curves please ignore the $n_{\mathrm{o}} \mu_{\mathrm{ISM}}$ factor.

For a mass scale we count total amount of gas material that the initial equilibrium disk had within a rectangular column of cross-section $\lambda_{y, \max } \times \lambda_{x, \max }$ extending vertically from $-z_{\mathrm{a}}$ to $+z_{\mathrm{a}}$. The wave number $\nu_{y}$, max of the maximum growth rate fixes azimuthal extent of the column cross-section. The undulation of field lines under self gravity introduces to the system a natural length scale in the azimuthal direction. However, it doesn't fix the radial extent, since the highest growth rate point in the dispersion-curve surface is always located on the $\nu_{y}$-axis. (see, for example, Figure 10.) Only scale we may call in for the radial extent is then the critical wavelength of marginal stability or the width of spiral arm. In our normalization scheme the critical wave number $\nu_{\text {crt }}$ becomes 1 or very close to it ( see Appendix $\mathrm{C}$ ), which gives a radial extent of $\lambda_{x, \max } \simeq 2 \pi H$. Since the total column density of halo-bound isothermal gas disk is $2 H \rho_{\mathrm{o}}(0) \tanh \zeta_{\mathrm{a}}$, we may have the mass scale, $\mathcal{M}_{\mathrm{crt}} \simeq 2 H n_{\mathrm{o}} \mu_{\mathrm{ISM}} m_{\mathrm{H}} \tanh \zeta_{\mathrm{a}} \cdot 2 \pi H \nu_{y, \max }^{-1} \cdot 2 \pi H$, as

$$
\mathcal{M}_{\mathrm{crt}} \simeq 1.4 \times 10^{7} \mathrm{M}_{\odot}\left[\frac{c_{\mathrm{s}}}{5 \mathrm{~km} \mathrm{~s}^{-1}}\right]^{3}\left[\frac{(1+\alpha)^{3}}{n_{\mathrm{o}} \mu_{\mathrm{ISM}} \mathrm{cm}^{3}}\right]^{1 / 2} \frac{1}{\nu_{y, \max }(\alpha)}
$$

When $\nu_{y, \max }^{\mathrm{ODD}}$ in Table 1 is substituted for $\nu_{y, \max }(\alpha)$ in the above equation, the mass scale varies with $\alpha$ as in Figure 13a, where the $n_{\mathrm{o}} \mu_{\mathrm{ISM}}$ factor is absorbed again in the ordinate. Since this mass scale depends on the velocity dispersion most sensitively, we have illustrated, in the figure, for the following four cases specifically: $c_{\mathrm{s}}=1,2,3$, and $5 \mathrm{~km} \mathrm{~s}^{-1}$.

Since the disk scale height is about $200 \mathrm{pc}$, the critical wavelength often turns out larger than the width of spiral arm, which is probably about a half kpc. For such cases, the arm width, $\Delta R$, should be taken for the radial extent of the rectangular column. The resulting mass scale, $\mathcal{M}_{\text {arm }}$, now reads

$$
\mathcal{M}_{\mathrm{arm}} \simeq 1.2 \times 10^{7} \mathrm{M}_{\odot}\left[\frac{c_{\mathrm{s}}}{5 \mathrm{~km} \mathrm{~s}^{-1}}\right]^{2}\left[\frac{\Delta R}{\mathrm{kpc}}\right]\left[\frac{1+\alpha}{\nu_{y, \max }(\alpha)}\right] .
$$

It is interesting to notice that once the arm width is fixed by the global dynamics of rotating disk, $\mathcal{M}_{\text {arm }}$ doesn't depend on the midplane density explicitly. Substituting $\nu_{y \text {, max }}^{\text {ODD }}$ for the wave number of the maximum growth rate, we have calculated the mass scale as a function of the magnetic-to-gas pressure ratio, and shown the results in Figure 13b for the four selected cases of the velocity dispersion. This figure was prepared with $\Delta R=1 \mathrm{kpc}$. 


\subsection{GMCs and HI Superclouds}

The two most prominent types of large interstellar structures are the GMCs (Dame et al. 1986; Blitz 1993) and the HI superclouds (Elmegreen \& Elmegreen 1987; Efremov \& Sitinik 1988; Elmegreen 1994). Clouds of each type show substantial spreads in mass, size, mean separation, internal velocity dispersion, and density. Particularly in the velocity dispersion the two types almost overlap with each other (Falgarone \& Lequeux 1973; Larson 1981; Heiles \& Troland 2003). However, the GMCs differentiate themselves as a distinct type from the HI superclouds in most of the directly observable properties (Alfaro et al. 1992). In Table 2 we have summarized observations of GMCs and HI superclouds separately. For entries of the GMCs we relied heavily on Blitz (1993), and the HI supercloud properties are mostly from Elmegreen \& Elmegreen (1987). The mean separation of the superclouds is known to depend on mass: The heavier are the clouds, the wider becomes their mean separation.

It is not easy to have a relevant time scale for structure formation directly from observations, but a number of estimates can still be made. For example, time required to pass an arm will serve a good criterion for the growth time, since gathering activity for the structure formation should take place mostly while the gas material stays in a spiral arm. As suggested by Kim \& Ostriker (2002), one half of the arm-to-arm moving time can be a reasonable measure for the arm-crossing period. In an $m$-armed spiral galaxy it will take $2 \pi R /\left[m R\left(\Omega-\Omega_{\mathrm{P}}\right)\right]$ for the gas material to complete a journey along the galactocentric circle of radius $R$ from one arm to the next. Here, $\Omega$ is the angular velocity of the galactic rotation at galactocentric distance $R$ and $\Omega_{\mathrm{P}}$ is that of the spiral pattern. With an approximation $\Omega_{\mathrm{P}} \simeq \Omega / 2$ (Binney \& Tremaine 1987) the arm-passing time simply becomes $4 \pi /(m \Omega)$. Keeping in mind the solar neighborhood in the Galaxy, we take $220 \mathrm{~km} \mathrm{~s}^{-1}$ for the rotation velocity at $R=8.5 \mathrm{kpc}$, and to the number of arms we may assign four (cf. Vallée 1995) to have

$$
\tau_{\mathrm{APT}} \simeq 60 \times 10^{6} \text { years }\left(\frac{4}{m}\right)\left[\frac{26 \mathrm{~km} \mathrm{~s}^{-1} \mathrm{kpc}^{-1}}{\Omega}\right] .
$$

Larson (1994) estimated the GMC growth time from the gas consumption rate in the Galaxy. The star formation rate is in the order of $3 \mathrm{M}_{\odot}$ per year, and about $2 \%$ of cloud mass is known to be converted into stars (Myers et al. 1986; Evans \& Lada 1991). This means that $150 \mathrm{M}_{\odot}$ of gas is being condensed, in every year, into star-forming molecular clouds. Since the total amount of gas in the Galaxy is about $5 \times 10^{9} \mathrm{M}_{\odot}$, mostly in the form

of GMCs, the time required to collect this much gas into the GMCs is about $33 \times 10^{6}$ years. One may then take $30 \times 10^{6}$ years as the formation time scale of the GMCs.

We now have three time-scale values: The arm-to-arm crossing period, $1.2 \times 10^{8}$ years, 
is thought be a somewhat generous time-scale criterion for the formation of HI superclouds, while the arm-passing time, $60 \times 10^{6}$ years, is a rather strict one for them. On the other hand, the gas consumption period, $30 \times 10^{6}$ years, will serve as a reasonable time-scale criterion for the formation of GMCs.

As is demonstrated in Figures 11, 12 and 13, the ranges of time, length and mass scales that are expected from the undular odd-parity solutions compare well with the observational criteria of the HI superclouds summarized in Table 2. To be specific, with the system parameter set, $n_{\mathrm{o}} \mu_{\mathrm{ISM}} \simeq 2 \mathrm{~cm}^{-3}, c_{\mathrm{S}} \simeq 5.8 \mathrm{~km} \mathrm{~s}^{-1}$ and $\alpha \simeq 0.5$, we have the growth time $\tau_{\mathrm{GRW}} \simeq$ $4.3 \times 10^{7}$ years, the mean separation $\Lambda \simeq 1.9 \mathrm{kpc}$, and the mass scale $\mathcal{M}_{\text {arm }} \simeq 2.8 \times 10^{7} \mathrm{M}_{\odot}$. With the same set, the disk scale height becomes $H \simeq 190 \mathrm{pc}$, and the resulting critical wavelength, $1.2 \mathrm{kpc}$, extends in the radial direction beyond the spiral arm. As illustrated in the figures, the choice of $\alpha=0.5$ won't make a critical issue in bounding the various scales; the scales depend least sensitively on $\alpha$, unless it is much bigger than unity. From the comparison we conclude that under canonical conditions of the Galactic ISM the Parker-Jeans instability can drive the ISM disk to form condensations of the HI supercloud scale.

To make the theoretical scales agree with the observational criteria of the GMCs, one has to strain the system parameters to extreme degrees. For example, a trial set of $n_{\mathrm{o}} \mu_{\mathrm{ISM}} \simeq$ $50 \mathrm{~cm}^{-3}, c_{\mathrm{s}} \simeq 1.2 \mathrm{~km} \mathrm{~s}^{-1}$ and $\alpha \simeq 1.0$ gives us 11 million years for the growth time, $510 \mathrm{pc}$ for the mean separation, and $\mathcal{M}_{\text {crt }} \simeq 1.4 \times 10^{5} \mathrm{M}_{\odot}$ for the mass scale of the GMCs. As done in this example, one could devise a combination of the system parameters that would formally satisfy the observational criteria of the GMCs. But the choice $n_{\mathrm{o}} \mu_{\mathrm{ISM}} \simeq 50 \mathrm{~cm}^{-3}$ seems too high to be the density of initial equilibrium configuration. And the choice of this high value of density requires the velocity dispersion of cloudlets too low. The undular odd-parity solutions under the self gravity alone may not grow the GMC and the HI supercloud scale structures simultaneously.

\section{DISCUSSION AND CONCLUSION}

Through the present study we come to realize that in previous investigations role of the interchange mode was unduly stressed in its tendency of disrupting large-scale ISM structures that the undular mode might otherwise have formed. The unfair emphasis was the price to

pay for both adoption of the external gravity as and neglect of the self gravity from driving agent of the Parker instability. In this paper we have examined what modifications could be made to the classical picture of the Parker instability by assigning the driving task solely to the self gravity. 
If the vertical acceleration rendered by the adopted driving agent is a constant of perpendicular distance from the central plane, it may act as a source of buoyancy force over the entire disk. Consequently, under such unrealistic uniform external gravity, convective motions are to be activated everywhere in the disk, and the interchange mode will indeed dominate the magnetized gas disk over the undular one. In reality, however, the vertical acceleration due mostly to the Galactic stars increases linearly, from zero at the midplane, with the perpendicular distance up to about a half kpc, beyond which it remains more or less constant. This type of external gravity can not be a buoyancy source in the disk midplane at least, where GMCs are mostly found; while the convection is pronouncedly active, under the realistic non-uniform gravity, only in high altitude regions, where the vertical acceleration reaches its maximum.

The convection wouldn't then be an obstacle for the classical Parker instability to form, for example, GMCs in the midplane, if the undular mode could collect enough material there before the interchange mode develops chaotic sheets at high altitudes. However, as long as the buoyancy force is available, the growth rate of the convection increases within the limiting value as the perturbation wavelength gets smaller and smaller. Therefore, even if all the realistic features are taken into account for the external gravity, the convection triggered by the interchange mode would develop small-scale features well before the classical Parker instability excited by the undular mode generates any dense large-scale structures in the disk central plane. Magnetic valleys eventually anchor some material towards the central plane, but the material takes form of vertically oriented thin sheets rather than of dense clumps, because the external gravity has only vertical component of acceleration. As long as the driving task is assigned to the external gravity, there is no means to divert the convective motions from the vertical direction and to form clumps instead of sheets.

The thick dashed-line in Figure 10 represents the growth rate contour corresponding to the highest ridge level. Any perturbations whose wave numbers are within the dashed-line boundary would grow faster than the ones outside. Therefore, with such perturbations, a collaboration of the Parker and Jeans instabilities may well suppress the convection and the disk will bear out large-scale structures.

Once the self gravity takes over the driving task from the external gravity, the effect of the Jeans gravitational instability due to the self gravity appears on the dispersion curves. The maximum growth rate of the Jeans instability at the large-wavelength perturbations can be be higher than the limiting growth rate of the convection at the small-wavelength perturbations, depending on the adiabatic index (cf., Figure 7). This is not what we've seen from the external gravity case. Therefore, for long-wavelength perturbations of odd-parity, the maximum growth rate of the undular mode becomes even faster than the highest growth 
rate value of the interchange mode (cf., Figure 10). The odd-parity undular mode thus opens up a route in the magnetized disk, through which the Parker and Jeans instabilities work jointly to form large-scale condensations in the midplane before the interchange mode disperses them into thin sheets.

In this way the Parker-Jeans instability is expected to remove major difficulties in forming the large-scale ISM structures. To check whether this expectation can be fulfilled by the odd-parity undular mode, we have compared the resulting time, length and mass scales with the corresponding observations of the HI superclouds and GMCs.

As far as the time scale is concerned, the Parker-Jeans instability grows quickly enough to satisfy the observational criteria for the HI superclouds and the GMCs as well (cf., Figure 11). The formation time scales inferred from various observations don't impose any tight limits on the system parameters, particularly on $\alpha$, and the comparison done in Figure 11 doesn't seem to differentiate the HI superclouds from the GMCs either. It was a bit surprise to us. Because of the neglect of cosmic rays, we thought, the time scale of the Parker-Jeans would meet the observational criteria only barely. In a recent study Ryu et al. (2003) treated cosmic rays with the diffusion-convection equation and realistic diffusion coefficients for both directions, perpendicular and parallel to the field lines. They have demonstrated that by pumping an extra buoyancy into the disk system, cosmic rays make an about factor two increase in the growth rate of the classical pure Parker mode, i.e., of the instability triggered by the undular perturbation under the uniform external gravity. The factor two may not be fully applied to the Parker-Jeans case, because the uniform external gravity exaggerates buoyancy. Nevertheless, if cosmic rays had been included, a substantial further reduction would have been made in the growth time scale. It may worth reminding that the Jeans gravitational instability was called in to support the Parker in the first place. And the result was successful, as was demonstrated by the $\left|\Omega_{\mathrm{y}, \max }^{\mathrm{ODD}}\right| /\left|\Omega_{\mathrm{y}, \max }^{\mathrm{EVN}}\right|-$ ratios in Table 1: A small increase in $\left|\Omega_{\mathrm{y}, \max }^{\mathrm{EVN}}\right|$ will result in a sizeable amplification through the ratio. An inclusion of rotation wouldn't change the situation in any significant way, because the rotation is known to decrease the growth rate by a few to fifteen percents only (Shu 1974). Along this line of reasonings, if $2 \mathrm{~cm}^{-3}$ is not an unrealistic choice for $n_{\mathrm{o}} \mu_{\mathrm{ISM}}$ in the Galaxy, we believe that the time scale we now have is an under-estimate for the Galactic ISM disk. The reason for the underestimation seems to be in our neglect of the external gravity.

As to the length scale, the Parker-Jeans instability seems to operate more efficiently than what is required for the HI superclouds. Under 'canonical' conditions of the Galactic ISM with $\alpha=0.5$, the odd-parity undular mode shows its maximum growth rate at wavelength $1.5 \mathrm{kpc}$, which is less than the observed mean separation, $2.4 \mathrm{kpc}$, of the HI superclouds (McGee \& Milton 1964; Elmegreen \& Elmegreen 1983; Alfaro et al. 1992; Efre- 
mov 1995). Because of the conceptual difficulty in applying a single velocity dispersion to the entire ISM disk, one may not consider this much difference in the length scale to be serious. However, we think the difference is a significant one, because existence of cosmic rays would reduce the wavelength further. Therefore, comparison of the length scale also suggests an over-subscription of the self gravity in our disk system.

From dispersion relations shown in Figures 1 and 2 we notice the growth rate peak for the even-parity undular mode doesn't survive in the competition with the odd-parity: The growth rate peak of the pure Parker mode is overwhelmed by the Parker-Jeans peak. This holds true for wide ranges of disk thickness and magnetic-to-gas pressure ratio. As was illustrated in Figure 3, the Parker peak may survive the Parker-Jeans overwhelming only in such unrealistically soft media as the ones having $\gamma \leq 0.7$, for example. In the magnetized gas disks where only the self gravity is operative to excite instabilities with the undular mode, it doesn't seem to be possible to have two distinct growth-rate peaks in the dispersion curve, and the present study indicates that the overwhelming Parker-Jeans maximum is strongly tied to the structures of the HI supercloud scale, not to the GMC scale ones. As Elmegreen(1994) pointed out, the characteristic mass and mean separation of the HI superclouds are natural outcomes of the gravitational instability in the self gravity dominating disk systems.

To relate both the GMC and HI supercloud structures to the single Parker-Jeans peak, one may rely on two-stage formation scenarios: The HI supercloud forms first and some time later the GMCs emerge as fragment products of the supercloud. This idea was suggested in the previous section reluctantly, because of the GMCs that are residing outside the supercloud. Almost all of the HI superclouds contain GMCs, but not all the GMCs belong to the HI superclouds (Elmegreen \& Elmegreen 1987; Efremov \& Sitnik 1988). Instead refining the two-stage idea, we should first pay attention to the external gravity heretofore ignored. In fact the external gravity renders vertical acceleration about 5 times stronger than the one expected from the equilibrium ISM stratification in our Galaxy (Bienaymé et al. 1987; Ferrière 1998a; Boulares \& Cox 1990; Lee et al. 2004). Of course in later stages the ever increasing self gravity of ISM eventually override the fixed external gravity, but in the initial stage the self gravity is definitely over-subscribed in our model disk. A balanced subscription between the external and self gravities for the task of instability driving would reduce dominance of the Parker-Jeans peak on one hand and enhance the Parker maximum on the other. In this way the odd-parity undular mode may exhibit two distinctly separated growth-rate peaks in the dispersion curve, with the one at smaller wave number corresponding to HI superclouds and the other at larger number to GMCs.

From these discussions we may conclude the paper as follows: The present study has 
reconfirmed that the Parker instability triggered by the undular mode under external gravity may not be a route towards the formation of such large-scale structures as the GMCs in the Galaxy (Kim et al. 2004). Even under the self gravity, the even-parity undular mode solution can not accommodate important observational properties of the large-scale structures; however, the odd-parity undular mode is shown to have all the potentials to remove major difficulties that have been confronting the classical Parker instability as the formation mechanism of the large-scale structures. The resulting time, length, and mass scales of the Parker-Jeans instability are shown to be in better agreement with the HI superclouds than with the GMCs. An inclusion of the external gravity along with a proper treatment of the cosmic rays would refine the agreement towards the desired direction.

It is our pleasure to acknowledge illuminating discussions and stimulating conversations with W.-T. Kim, B. G. Elmegreen, J. Kim, D. Ryu, and J. Franco. The authors are grateful to B.-C. Koo and T. Nakagawa for guiding us to some important literatures. We are also thankful to J. Pyo for his assistance in the preparation of some of the diagrams. While preparing this paper, SSH was benefited greatly from Astronomical Information Research Group of the KASI, Korea and also from Laboratory Infrared Astrophysics of the ISAS/JAXA, Japan. This study was supported in part by KOSEF through grant R14-2002-058-01003-0. 


\section{APPENDIX}

\section{A. Coefficients of Perturbation Equations}

The coeffients of the two second order ODEs [Eqs. (24) and (25)] are as follow:

$$
\begin{gathered}
A_{2} \equiv\left(\Omega^{2}-\frac{2 \alpha}{1+\alpha} \eta^{2}\right)\left\{\frac{2 \alpha+\gamma}{1+\alpha} \Omega^{2}-\frac{2 \alpha \gamma}{(1+\alpha)^{2}} \eta^{2}\right\} \\
A_{1} \equiv-2 \Theta\left(\Omega^{2}-\frac{2 \alpha}{1+\alpha} \eta^{2}\right)\left\{\frac{2 \alpha+\gamma}{1+\alpha} \Omega^{2}-\frac{2 \alpha \gamma}{(1+\alpha)^{2}} \eta^{2}\right\} \\
A_{0} \equiv-2\left(1-\Theta^{2}\right) \Omega^{4}+\frac{4}{1+\alpha}\left[\alpha\left(1-\Theta^{2}\right) \eta^{2}-\left\{(1-\alpha-\gamma) \xi^{2}+(1+\alpha-\gamma) \eta^{2}\right\} \Theta^{2}\right] \Omega^{2} \\
+\frac{8 \alpha(1+\alpha-\gamma)}{(1+\alpha)^{2}} \Theta^{2} \eta^{2} k^{2}+\left(\Omega^{2}-\frac{2 \alpha}{1+\alpha} \eta^{2}\right)\left\{\Omega^{4}-\frac{2 \alpha+\gamma}{1+\alpha} k^{2} \Omega^{2}+\frac{2 \alpha \gamma}{(1+\alpha)^{2}} \eta^{2} k^{2}\right\} \\
\left.B_{0} \equiv-i \Omega\left[2 \frac{1+\alpha-\gamma}{1+\alpha} k^{2}-4 \frac{\alpha}{1+\alpha} \xi^{2}\right\} \Theta \Omega^{2}-\frac{4 \alpha(1+\alpha-\gamma)}{(1+\alpha)^{2}} \Theta \eta^{2} k^{2}\right] \\
C_{2} \equiv i \Omega\left\{\Omega^{4}-\frac{2 \alpha+\gamma}{1+\alpha} k^{2} \Omega^{2}+\frac{2 \alpha \gamma}{(1+\alpha)^{2}} \eta^{2} k^{2}\right\} \\
C_{0} \equiv-k^{2} i \Omega\left[\Omega^{4}+\left\{2\left(1-\Theta^{2}\right)-\frac{2 \alpha+\gamma}{1+\alpha} k^{2}\right\} \Omega^{2}+\frac{2 \alpha}{1+\alpha}\left\{\frac{\gamma}{1+\alpha} k^{2}-2\left(1-\Theta^{2}\right)\right\} \eta^{2}\right] \\
D_{1} \equiv 2\left(1-\Theta^{2}\right) \Omega^{2}\left(\Omega^{2}-\frac{2 \alpha}{1+\alpha} \eta^{2}\right)
\end{gathered}
$$

and

$$
D_{0} \equiv-4 \Theta\left(1-\Theta^{2}\right)\left\{\Omega^{4}+\frac{1-\alpha-\gamma}{1+\alpha} k^{2} \Omega^{2}-\frac{2 \alpha(1+\alpha-\gamma)}{(1+\alpha)^{2}} \eta^{2} k^{2}\right\} .
$$

Here $k^{2}$ means $\xi^{2}+\eta^{2}$, and the other symbols have the same meanings as in $\S 2$ of the main text.

\section{B. Stable Modes}

In self-gravitating, magnetized, gaseous disks, we expect to have such stable wave modes as gravity wave, Alfvén wave, and fast and slow MHD waves. To check whether our system of the linearized perturbation equations indeed has all these modes, we take the equations in various limits of perturbation wave numbers. 


\section{B.1. Vertical Gravity Wave Mode}

The vertical gravity mode can be isolated by taking the perturbation equations in the limit $\nu_{x}=0$ and $\nu_{y}=0$. This wave travels vertically and is related to convective motions (Lamb 1945).

We eliminate $\psi$ from equations (24) and (25) and put $\nu_{x}=0$ and $\nu_{y}=0$ in the resulting equation for $u_{z}$ to have

$$
\frac{d^{3}}{d \zeta^{3}} u_{z}-2 \frac{d}{d \zeta}\left[\Theta \frac{d}{d \zeta} u_{z}\right]-\frac{1+\alpha}{2 \alpha+\gamma} \Omega^{2} \frac{d}{d \zeta} u_{z}=0
$$

Since for odd-parity solutions

$$
\left.\frac{d^{2}}{d \zeta^{2}} u_{z}\right|_{\zeta=0}=0 \quad \text { and }\left.\quad u_{z}\right|_{\zeta=0}=0
$$

hold true, we may integrate equation (B1) and have

$$
\frac{d^{2}}{d \zeta^{2}} u_{z}-2 \Theta \frac{d}{d \zeta} u_{z}-\frac{1+\alpha}{2 \alpha+\gamma} \Omega^{2} u_{z}=0
$$

Changing variable $\zeta$ to $Z \equiv \tanh \zeta$, we rewrites this as

$$
\left(1-Z^{2}\right)^{2} \frac{d^{2}}{d Z^{2}} u_{z}-4 Z\left(1-Z^{2}\right) \frac{d}{d Z} u_{z}-\sigma^{2} u_{z}=0
$$

where $\sigma$ is defined as

$$
\sigma^{2} \equiv \frac{1+\alpha}{2 \alpha+\gamma} \Omega^{2}
$$

We then substitute $D=\left(1-Z^{2}\right)^{-p} u_{z}(Z)$ for $u_{z}(Z)$ and $\varpi=(1+Z) / 2$ for $Z$. With parameter $p$ defined by

$$
\sigma^{2}=4 p(p-1 / 2)
$$

Equation (B4) reads

$$
\varpi(1-\varpi) \frac{d^{2}}{d \varpi^{2}} D+\{2 p+1 / 2-(4 p+1) \varpi\} \frac{d}{d \varpi} D-4 p^{2} D=0,
$$

which is a hypergeometric differential equation. In terms of $Z$ its general solution is given by

$$
\begin{aligned}
u_{z}(Z) & =\left(1-Z^{2}\right)^{p}\left[C_{1} \mathrm{~F}\{2 p, 2 p ; 2 p+1 / 2 ; 1 / 2(1+Z)\}\right. \\
& \left.+C_{2}\{1 / 2(1+Z)\}^{1 / 2-2 p} F\{1 / 2,1 / 2 ; 3 / 2-2 p ; 1 / 2(1+Z)\}\right]
\end{aligned}
$$


where $\mathrm{F}$ is a hypergeometric function, and $C_{1}$ and $C_{2}$ are integral constants (Polyanin \& Zaitsev 1995).

From the boundary conditions (Eq. [B2]), we construct a set of two simultaneous indefinite equations of $C_{1}$ and $C_{2}$. To have nontrivial solutions for the integral constants, matrix of the simultaneous equations should have vanishing determinant $G(p)$. This condition gives us secular equation $G(p)=0$ with the following definition of $G(p)$ :

$$
\begin{aligned}
G(p) & =\frac{p^{2}(2 p+1)^{2}}{(2 p+1 / 2)(2 p+3 / 2)} \mathrm{F}(1 / 2,1 / 2 ; 3 / 2-2 p ; 1 / 2) \mathrm{F}(2 p+2,2 p+2 ; 2 p+5 / 2 ; 1 / 2) \\
& -\left[\left(4 p^{2}-1 / 4\right) \mathrm{F}(1 / 2,1 / 2 ; 3 / 2-2 p ; 1 / 2)+\frac{2 p-1 / 2}{2(4 p-3)} \mathrm{F}(3 / 2,3 / 2 ; 5 / 2-2 p ; 1 / 2)(\mathrm{B} 9)\right. \\
& \left.+\frac{9}{8(4 p-3)(4 p-5)} \mathrm{F}(5 / 2,5 / 2 ; 7 / 2-2 p ; 1 / 2)\right] \mathrm{F}(2 p, 2 p ; 2 p+1 / 2 ; 1 / 2) .
\end{aligned}
$$

In Figure A1 we have plotted $G(p)$ against $p$. As can be seen from the figure, $G(p)$ becomes zero at negative half integers and also at zero. In terms of $p$ the resulting eigenfrequencies $\Omega$ are given as

$$
\Omega^{2}=\frac{2 \alpha+\gamma}{1+\alpha} 2 p(2 p-1)
$$

where $p=-3 k(2 k-1) / 2$ with $k=0,1,2, \cdots$.

This mode of stable waves represents gravity waves propagating vertically in the equilibrium disk ( cf., Eq. [50] in Shu 1974). In the absence of magnetic fields Lamb (1945) called this as the atmospheric wave. This mode appears at the very beginning stage of nonlinear MHD simulations.

\section{B.2. Fast MHD Wave}

For the limiting case $\xi \neq 0$ and $\eta=0$, perturbations in the $x$-direction disturb density, pressure and magnetic fields in such a way that the field strength and density show alternating regions of compression and rarefaction. This is a signature of simple acoustic waves. However, it is not easy to isolate this type of acoustic waves near the dense midplane, where the selfgravitational force is likely to override the restoring force of the magnetic pressure. In order to isolate the mode clearly, we apply the equation to the limit $z \rightarrow \infty$, where the gravity force becomes very weak.

In this limit $(\xi \neq 0, \eta=0, z \rightarrow \infty)$ one may easily isolate the fast MHD wave mode,

$$
\Omega^{2}=\frac{2 \alpha+\gamma}{1+\alpha} \xi^{2}
$$


or

$$
\omega^{2}=\left(v_{\mathrm{A}}^{2}+a_{\mathrm{s}}^{2}\right) k_{x}^{2},
$$

where $a_{\mathrm{s}}^{2}$ means $\gamma c_{\mathrm{s}}^{2}$. The numerator $2 \alpha+\gamma$ of equation (B12) indicates a coupling of Alfvén and sound waves. And the $\xi^{2}$-term suggests that the wave propagates in the horizontal direction perpendicularly to the initial magnetic fields. The denominator $1+\alpha$ comes from the definition of the scale height, $H$.

\section{B.3. Alfvén and Slow MHD Waves}

To isolate the Alfvén and the slow MHD modes, it is better to suppress the Jeans and the Parker instabilities. We thus push the perturbation equations to the limit $1 \ll \eta \ll \xi$, and have the following dispersion relation:

$$
(2 \alpha+\gamma) \Omega^{4}-\frac{4 \alpha(\alpha+\gamma)}{1+\alpha} \eta^{2} \Omega^{2}+\frac{4 \alpha^{2} \gamma}{(1+\alpha)^{2}} \eta^{2}=0 .
$$

This can be decoupled into the Alfvén mode

$$
\Omega^{2}=\frac{2 \alpha}{1+\alpha} \eta^{2}, \quad \text { or } \quad \omega^{2}=v_{\mathrm{A}}^{2} k_{y}^{2}
$$

and the slow mode of the MHD waves

$$
\Omega^{2}=\frac{2 \alpha \gamma}{(1+\alpha)(2 \alpha+\gamma)} \eta^{2}, \quad \text { or } \quad \omega^{2}=\frac{v_{\mathrm{A}}^{2} a_{s}^{2}}{v_{\mathrm{A}}^{2}+a_{s}^{2}} k_{y}{ }^{2} .
$$

The two waves propagate in the horizontal plane along the direction of initial magnetic fields. The $2 \alpha \gamma /(2 \alpha+\gamma)$-term represents the cusp speed for the group velocity of the slow wave.

Finally, the magnetized sound wave is derived from the $y$-component of the momentum equation. The sound waves propagate along the magnetic field direction, with the dispersion relation given by

$$
\Omega^{2}=\frac{\gamma}{1+\alpha} \eta^{2}, \quad \text { or } \quad \omega^{2}=a_{s}{ }^{2} k_{y}{ }^{2} .
$$

\section{Marginal Stability Analysis}

For thick disks the Parker instability may assist the Jeans gravitational instability, so the maximum of the growth rate curve is located on the $\nu_{y}$-axis in the plane of perturbation wave numbers. This was demonstrated in Figures 9c, 9d and 10, where the peak on the $\nu_{y}$-axis is higher than the one on the $\nu_{x}$-axis. As the disk thickness decreases, the growth 
rate peak on the $\nu_{y}$-axis decreases, since the Parker instability is harder to get triggered in thinner disks. In the disk of the critical thickness, the Parker instability disappears, and the two peaks on the $\nu_{x^{-}}$and $\nu_{y}$-axes become of the same height. For the disk thinner than the critical thickness, the magnetic fields obstruct the system from developing the Jeans instability along the $y$-direction. So, the maximum of the growth rate curve is now located on the $\nu_{x}$-axis.

As was shown in Figure 3, the dispersion relation of the Jeans-Parker instability has two local maxima. The peak at smaller wave number is for the Jeans instability, while the one at larger number for the Parker instability. The dispersion curve crosses a critical wave number, which separates the region of stability from that of instability. The critical wave number comes into being due to magnetic tension, and the Parker instability gets stabilized by the tension. Thus, investigating the marginal state, we can find the critical thickness for the disk that may trigger the Parker instability.

With $\xi=0$ and $\Omega=0$, equation (21) describes the marginal state of the Parker instability as

$$
\frac{\mathrm{d}^{2}}{\mathrm{~d} \zeta^{2}} u_{z}-2 \Theta \frac{\mathrm{d}}{\mathrm{d} \zeta} u_{z}+\left[\frac{2(1+\alpha-\gamma)(1+\alpha)}{\alpha \gamma} \Theta^{2}-\eta^{2}\right] u_{z}=0
$$

where all the symbols have their usual meanings. Applying equation (C1) to the disk boundary and denoting the eigenvalue of the equation by

$$
E V=\frac{2(1+\alpha-\gamma)(1+\alpha)}{\alpha \gamma} \Theta_{a}^{2}-\eta^{2}
$$

where $\Theta_{\mathrm{a}}=\tanh \zeta_{\mathrm{a}}$, we have the critical wavelength as

$$
\lambda_{\mathrm{crt}}=2 \pi\left[\frac{(1+\alpha) c_{\mathrm{s}}^{2}}{2 \pi \mathrm{G} \rho_{\mathrm{o}}(0)}\right]^{1 / 2}\left[\frac{2(1+\alpha-\gamma)(1+\alpha)}{\alpha \gamma} \Theta_{\mathrm{a}}^{2}-E V\right]^{-1 / 2}
$$

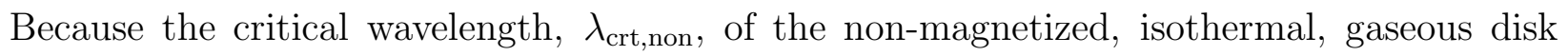
which is infinitely extended in vertical direction is given by

$$
\lambda_{\text {crt,non }}=2 \pi \sqrt{\frac{c_{s}^{2}}{2 \pi G \rho_{\mathrm{o}}(0)}},
$$

we know, equation (C3) should reduce to Equation (C4), in the limit $\alpha \rightarrow 0, \gamma=1$ and $\Theta_{a}$ $=1$. This can be realized by simply assigning unity to $E V$. From this we infer $E V=1$ is an eigenvalue of our halo-bound finite disk. Therefore, we may have the normalized critical wave number of the perturbation propagating along the magnetic fields as

$$
\eta_{\text {crt }}=\left[\frac{2(1+\alpha-\gamma)(1+\alpha)}{\alpha \gamma} \Theta_{a}^{2}-1\right]^{1 / 2} .
$$


This nicely recovers the critical wave number, $\sqrt{2 \alpha+1}$, of a magnetized, isothermal, gaseous disk infinitely extended in vertical direction (Stodólkiewicz 1963).

Since the Parker instability may not be triggered in a disk whose critical wave number is zero, from equation (C5), we have the critical adiabatic index for an onset of the Parker instability as:

$$
\gamma_{\mathrm{crt}}=\frac{(1+\alpha)^{2} \Theta_{a}^{2}}{\Theta^{2}+\frac{1}{2}\left(1+2 \Theta_{a}^{2}\right) \alpha}
$$

which again recovers the well-known $\gamma_{\mathrm{crt}}=(1+\alpha)^{2} /(1+3 \alpha / 2)$ condition for the classical Parker instability in an infinitely extended disk under external gravity (Kim \& Hong 1998). In the disk with $\gamma<\gamma_{\text {crt }}$, we won't expect the Parker instability to take a place. In Figure 3 we have illustrated how the material rigidity controls the dispersion relation of the ParkerJeans instability. The dispersion curve usually shows two bumps, one from the Jeans and the other the Parker. But the Parker bump becomes inconspicuous as the adiabatic index increase, the even-parity dispersion curve in dashed line almost coincides with the wave number axis when $\gamma=1.04$, and completely disappears as $\gamma$ further increases; from equation (C6) we indeed have $\gamma_{\mathrm{crt}}=1.05$ with $\alpha=0.1$ and $\zeta_{\mathrm{a}}=5$.

In $§ 3.1$, we have noticed that the Parker instability may assist the Jeans instability only in thick enough disks; thin disks may not conceive the Parker instability in the first place. What is then the critical thickness separating the thin from thick disk? This can be answered from equation (C5) by finding out such $\Theta_{a}\left(=\tanh \zeta_{a}\right)$ that may make $\eta_{\text {crt }}$ zero, namely,

$$
\zeta_{\mathrm{a}, \mathrm{crt}}=\tanh ^{-1}\left[\frac{\alpha \gamma}{2(1+\alpha-\gamma)(1+\alpha)}\right]^{1 / 2} \text {. }
$$

When $\zeta_{a}<\zeta_{a, \text { crt }}$, the Parker instability cannot operate in the system, and the magnetic fields instead obstruct the self-gravitational instability. Maximum of the growth rate curve for the gravitational instability is located, in this case, on the $\nu_{x}$-axis, so the gravitational instability would generate a long cylinder whose axis is parallel to the magnetic field lines. On the other hand, when $\zeta_{a}>\zeta_{a, \text { crt }}$, the Parker instability assists the gravitational instability, and the Parker-Jeans instability is operative in the system. Maximum of the growth rate curve for the Jeans-Parker instability is placed now on the $\nu_{y}$-axis, so the instability would align the cylinder axis perpendicularly to the field lines. When $\zeta_{a}=\zeta_{a, \text { crt }}$, the instability takes place, and the Parker instability cannot assist the Jeans to grow faster along the fields. Thereby, the perturbation propagating along the $y$-direction coordinates with that propagating along the $x$-direction. The critical thickness, when $\alpha=1.0$ and $\gamma=1.0$ for example, becomes $0.549 \mathrm{H}$. As shown in Figure A2, the undular mode $\left(\nu_{x}=0\right)$ dispersion curve in solid line looks almost the same as the interchange mode $\left(\nu_{y}=0\right)$ one in dashed line. 
Table 1. Comparison of the Odd- and Even-Parity Solutions

\begin{tabular}{ccccccc}
\hline \hline$\alpha$ & $\left|\Omega_{\mathrm{y}, \max }^{\mathrm{ODD}}\right|$ & $\left|\Omega_{\mathrm{y}, \max }^{\mathrm{EVN}}\right|$ & $\tau_{\min }^{\mathrm{ODD}} / \tau_{\min }^{\mathrm{EVN}}$ & $\nu_{\mathrm{y}, \max }^{\mathrm{ODD}}$ & $\nu_{\mathrm{y}, \max }^{\mathrm{EVN}}$ & $\lambda_{\mathrm{y}, \max }^{\mathrm{ODD}} / \lambda_{\mathrm{y}, \max }^{\mathrm{EVN}}$ \\
\hline 0.00 & 0.665 & - & - & 0.476 & - & - \\
0.25 & 0.722 & 0.259 & 0.359 & 0.547 & 0.750 & 1.371 \\
0.50 & 0.766 & 0.372 & 0.486 & 0.621 & 0.838 & 1.350 \\
1.00 & 0.835 & 0.513 & 0.614 & 0.749 & 1.000 & 1.334 \\
2.00 & 0.924 & 0.675 & 0.730 & 0.943 & 1.239 & 1.313 \\
3.00 & 0.981 & 0.765 & 0.780 & 1.093 & 1.417 & 1.296 \\
\hline
\end{tabular}


Table 2. Observational Properties of GMCs and HI superclouds

\begin{tabular}{lcc}
\hline \hline \multicolumn{1}{c}{ Properties } & GMCs & HI Superclouds \\
\hline Mass [M $\odot$ ] & $(1 \sim 10) \times 10^{5 \mathrm{a}, \mathrm{b}}$ & $(1 \sim 40) \times 10^{6 \mathrm{c}}$ \\
Mean Separation [kpc] & $0.4 \sim 0.6^{\mathrm{d}}$ & $\sim 2.4^{\mathrm{e}}$ \\
Velocity Dispersion [km s$\left.{ }^{-1}\right]$ & $0.4 \sim 8.5^{\mathrm{f}, \mathrm{g}}$ & $1.1 \sim 8.1^{\mathrm{a}}$ \\
Mean Density $\left[\mathrm{cm}^{-3}\right]$ & $\sim 10^{2} \mathrm{H}_{2}{ }^{\mathrm{d}}$ & $\gtrsim 9 \mathrm{H}^{\mathrm{c}}$ \\
Size [pc] & $\sim 45^{\mathrm{d}}$ & $\sim 400^{\mathrm{c}}$ \\
formation time scale [years] & $30 \times 10^{6}$ & $80 \times 10^{6}$ \\
\hline
\end{tabular}

${ }^{a}$ arson 1981

${ }^{\mathrm{b}}$ Dame et al. 1986

'Elmegreen \& Elmegreen 1987

${ }^{\mathrm{d} B l i t z} 1993$

eAlfaro et al. 1992

${ }^{\mathrm{f}}$ Heiles \& Troland 2003

${ }^{g}$ Falgarone \& Lequeux 1973 


\section{REFERENCES}

Alfaro, E. J., Cabrera-Caño, J., \& Delgado, A, J. 1992, ApJ, 399, 576

Appenzellar, I. 1974, A\&A, 36, 99

Asséo, E., Cesarsky, C. J., Lachièze-Rey, M., \& Pellat, R. 1978, ApJ, 225, L21

Bienaymé, O., Robin, A. C., \& Crézé, M. 1987, A\&A, 180, 94

Binney, J., \& Tremaine, S. 1987, in Galactic Dynamics, (Princeton: Princeton Univ. Pres.), 386

Blitz, L. 1993, in Physics of Star Formation and Early Stellar Evolution, ed. C. J. Lada, N. D. Kylafis, \& G. Meynet (Dordrecht: Kluwer), 3

Blitz, L., \& Shu, F. H. 1980, ApJ, 238, 148

Boulares, A. \& Cox, D. P. 1990, ApJ, 365, 544

Chandrasekhar, S. 1961, in Hydrodynamics and Hydromagnetic Stability (Oxford: Clarendon Press), 457

Chandrasekhar, S., \& Fermi, E. 1953, ApJ, 118, 116

Chou, W., Matsumoto, R., Tajima, T., Umekawa, M., \& Shibata, K. 2000, ApJ, 538, 710

Dame, T. M., Elmegreen, B. G., Cohen, R. S., \& Thaddeus, P. 1986, ApJ, 305, 892

Efremov, Y. N. 1995, AJ, 110, 2757

Efremov, Y. N., \& Sitnik, T. G. 1988, Pi'ma Astron. Zh., 14, 817

Elmegreen, B. G. 1982, ApJ, 253, 634

-. 1994, ApJ, 433, 39

Elmegreen, B. G. \& Elmegreen, D. M. 1978, ApJ, 220, 1051

- 1983, MNRAS, 203, 31

- 1987, ApJ, 320, 182

Evans, N. J., \& Lada, E. A. 1991, in Star Formation in Three Nearby Molecular Cloud Complexes, eds., E. Falarone, F. Boulanger, \& G. Duvert, (Dordrecht: Kluwer Aca. Pub.), 293 
Falgarone, E., \& Lequeux, J. 1973, A\&A, 25, 253

Ferrière, K. M. 1995, ApJ, 441, 281

—. 1998a, ApJ, 487, 759

—. 1998b, ApJ, 503, 700

Franco, J., Kim, J., Alaro, E. J., \& Hong, S. S. 2002, ApJ, 570, 647

Gilman, P. A. 1970, ApJ, 162, 1019

Giz, A. T., \& Shu, F. H. 1993, ApJ, 404, 185

Goldreich, P., \& Lynden-Bell, D. 1965, MNRAS, 130, 125

Handa, T., Sofue, Y., Ikeuchi, S., Kawabe, R., \& Ishizuki, S. 1992, PASJ, 44, L227

Holmberg, J. \& Flynn, C. 2000, MNRAS, 313,209

Heiles, C. \& Troland, T. H. 2003, ApJ, 586, 1067

Hong, S. S. \& Kim, J. 1997, in The Physical Processes in the Interstellar Medium, (Min Eum: Seoul), 263

Hughes, D. W., \& Procter, M. R. E. 1988, Ann. Rev. Fluid Mech., 20, 187

Kim, J., \& Hong, S. S. 1998, ApJ, 507, 254

Kim, J., Ryu, D., Jones, T. W., \& Hong, S. S. 1998, ApJ, 506, L139

Kim, J., Ryu, D., Hong, S. S. 2004, Ap\&SS, 292, 255

Kim, J., Franco, J., Hong, S. S., Santillán, A., \& Martos, M. A. 2000, ApJ, 531, 873

Kim, W.-T., \& Ostriker, E. C. 2002, ApJ, 570, 652

Kulkarni, S. R., \& Heiles, C. 1987, in Interstellar Processes, ed. D. J. Hollenbach, \& H. A. Thronson, Jr. (Dordrecht: Reidel), 87

Lachièze-Rey, M., Asséo, e., Cesarsky, C. J., Pellat, R. 1980, ApJ, 315, 175

Lamb, H. 1945, in Hydrodynamics, (Cambridge: Univ. of Cambridge), 429

Larson, R. B. 1981, MNRAS, 194, 809 
Larson, R. B. 1994, in The Structure and Content of Molecular Clouds: 25 Years of Molecular Radioastronomy, proceedings of a conference at Ringberg Castle, ed. T. L. Wilson \& K. J. Johnston (Springer-Verlag: Berlin), 13

Lee, S. M. 1993, Master Thesis, Seoul National University

—. 2002, Ph.D. Thesis, Seoul National University

Lee, S. M. \& Hong, S. S., 1999, in Proceedings of the 4th East Asian Meeting on Astronomy, 293

Lee, S. M., Kim, J., Franco, J., \& Hong, S. S. 2004, J. Korean Astron. Soc., 37, 249

Lubow, S. H. \& Pringle, J. E. 1993, MNRAS, 263, 701

McGee, R. X., Milton, J. A. 1964, Aust. J. Phys., 17, 128

Mouschovias, T. Ch. 1974, ApJ, 192, 37

Mouschovias, T. Ch., Shu, F. H., \& Woodward, P. R. 1974, A\&A, 33, 73

Myers, P. C., Dame, T. M., Thaddeus, P., Cohen, R. S., Silverberg, R. F., Dwek, E., \& Hanser, M. G. 1986, ApJ, 301,398

Nagai, T., Inutsuka, S., \& Miyama, S. M. 1998, ApJ, 506, 306

Nakamura, F., Hanawa, T., \& Nakano, T. 1991, PASJ, 43, 685

Nakano, T. 1988, PASJ, 40, 593

Newcomb, W. A. 1961, Phys. Fluids, 4, 391

Parker, E. N. 1955, ApJ, 121, 491

- 1966, ApJ, 145, 811

- 1967, ApJ, 149, 535

Polyanin, A. D. \& Zaitsev, V. F. 1995, in Handbook of Exact Solutions for Ordinary Differential Equations (Boca Raton: CRC Press), 152

Ryu, D., Kim, J., Hong, S. S. \& Jones, T. W. 2003, ApJ, 589, 338

Shu, F. 1974, A\&A, 33, 55

Shibata, K., \& Matsumoto, R. 1991, Nature, 353, 633 
Simon, R. 1965, Ann. d'Ap., 28, 40

Spitzer, L., Jr. 1978, in Physical Processes in the Interstellar Medium (New York: Wiley), 226

Stodólkiewicz, J. S. 1963, Acta Astro., 13, 30

Tomisaka, K., \& Ikeuchi, S. 1983, PASJ, 35, 187

Vallée, J. P. 1995, A\&A, 301, 11

Zweibel, E. G., \& Kulsrud, R. M. 1975, ApJ, 201, 63 

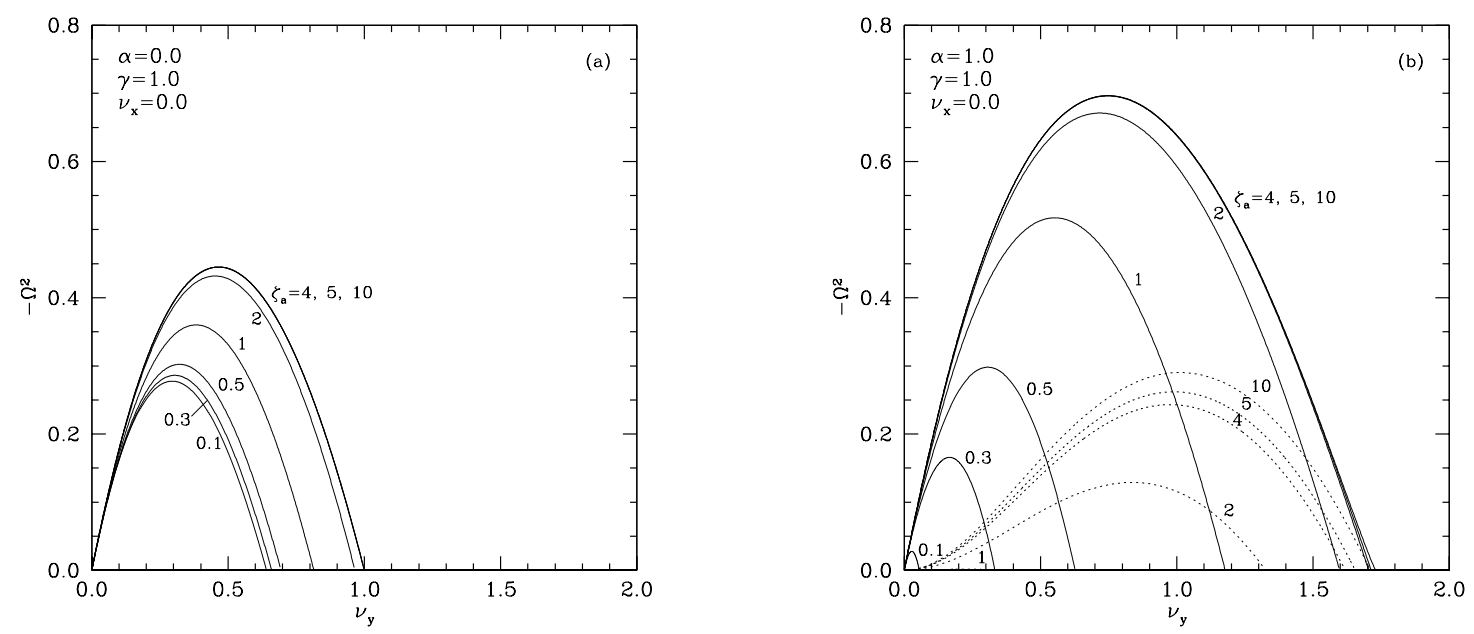

Fig. 1.- Dispersion relation of the undular mode $\left(\nu_{x}=0\right)$ for $(a)$ non-magnetized and $(b)$ magnetized gaseous discs of various thickness. The abscissa denotes effective wave number $\nu_{y}$, and the ordinate does square of the growth rate. Some of the disk and perturbation parameters are given in the upper left corner of each frame. The solid and dashed lines in (b) are for odd- and even-parity solutions, respectively. Because even-parity can not activate gravitational instability, there is marked suppression in the maximum growth rate from the odd- to the even-parity solutions. 


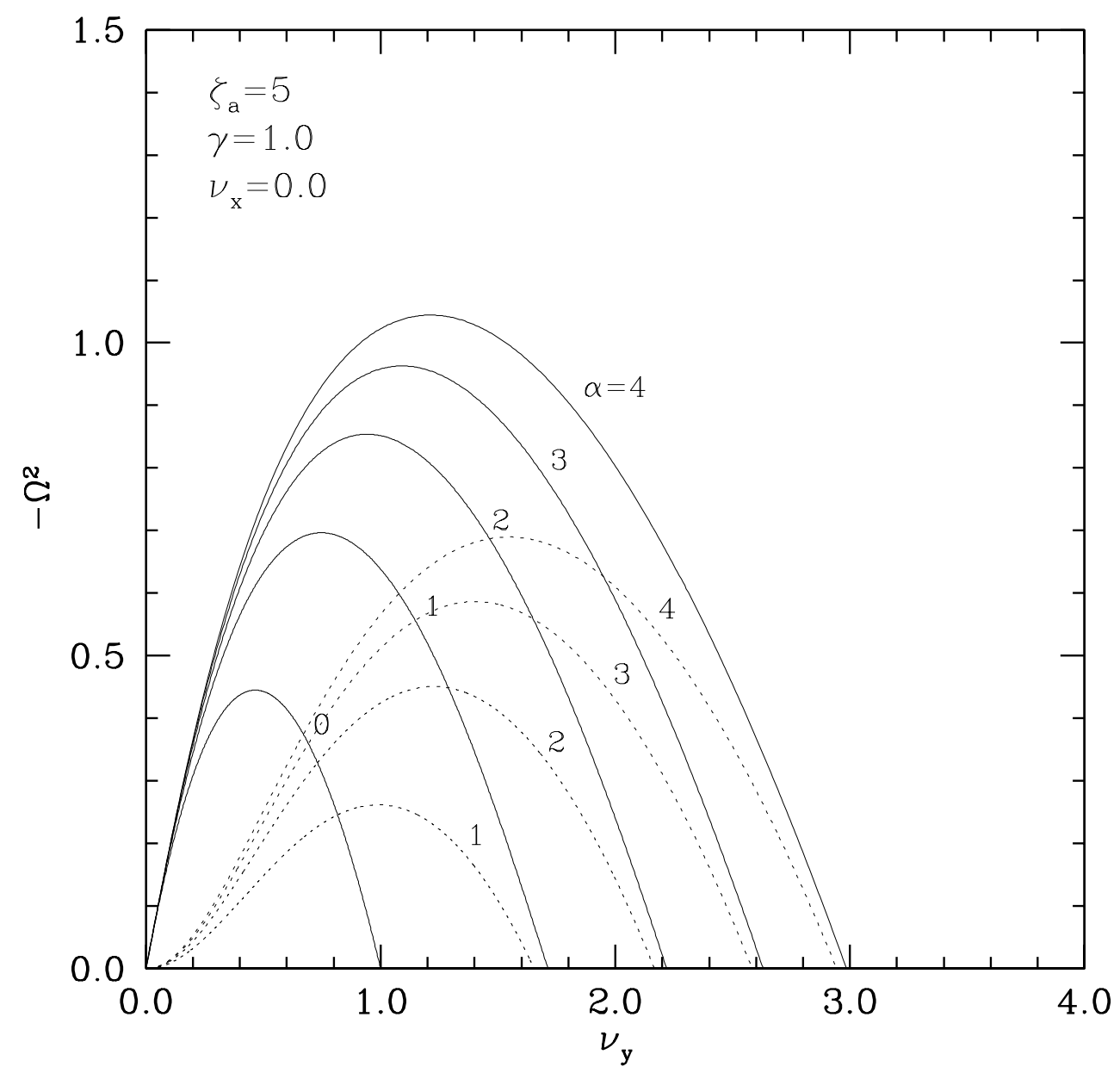

Fig. 2.- Dispersion relation of the undular mode $\left(\nu_{x}=0\right)$ for various values of $\alpha$. The abscissa denotes effective wave number $\nu_{y}$, and the ordinate does square of the growth rate. Some of the disk and perturbation parameters are given in the upper left corner. The solid and dashed lines are for odd- and even-parity solutions, respectively. 


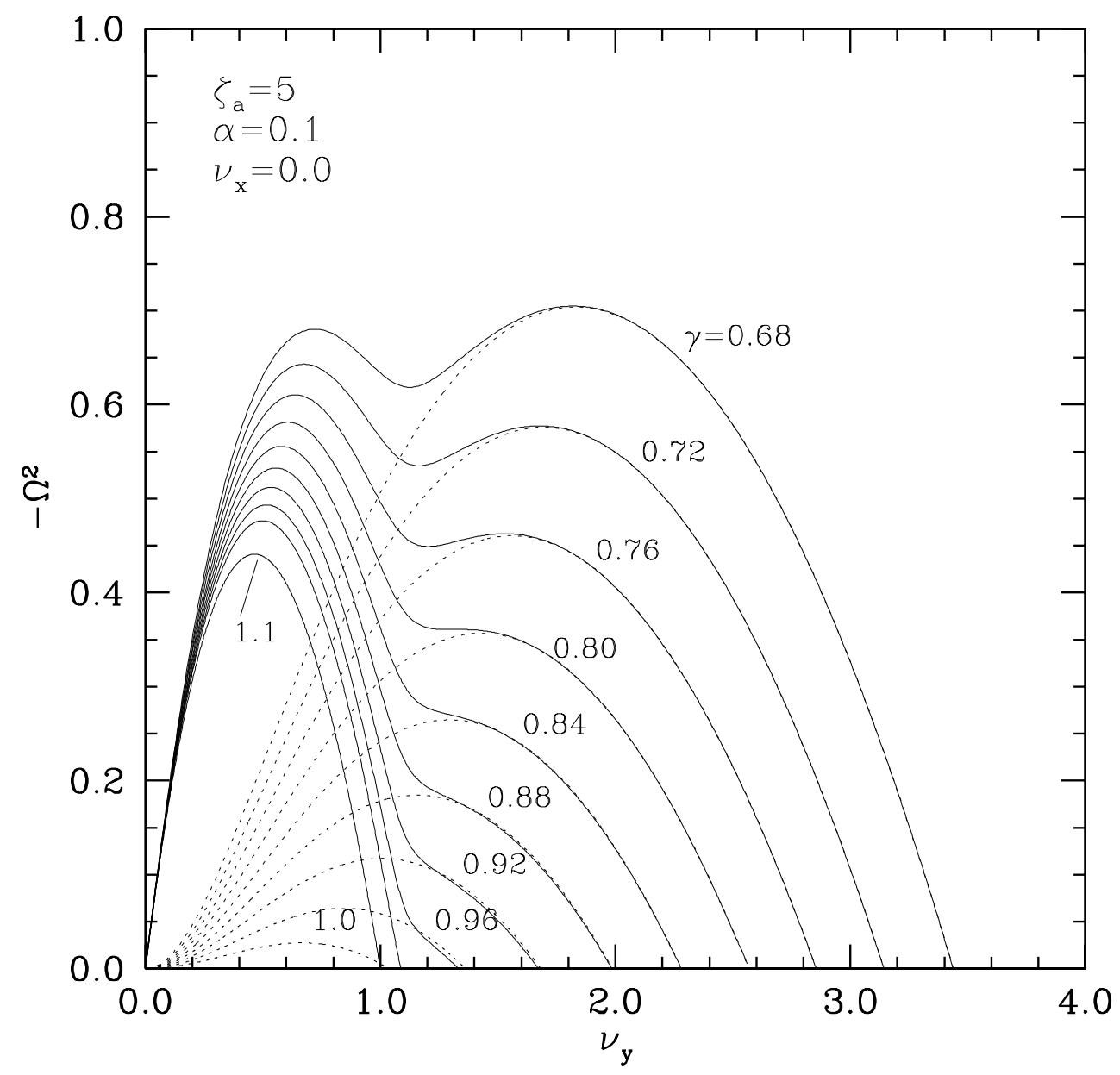

Fig. 3.- Dispersion relations for selected values of $\gamma$. The abscissa denotes effective wave number $\nu_{y}$ and the ordinate does square of the normalized growth rate of undular mode perturbation $\left(\nu_{x}=0.0\right)$. Solid and dashed lines are for the odd- and even-parity solutions, respectively. The disk parameters are specified in the upper left corner, and $\gamma$-values are marked to each curve. 

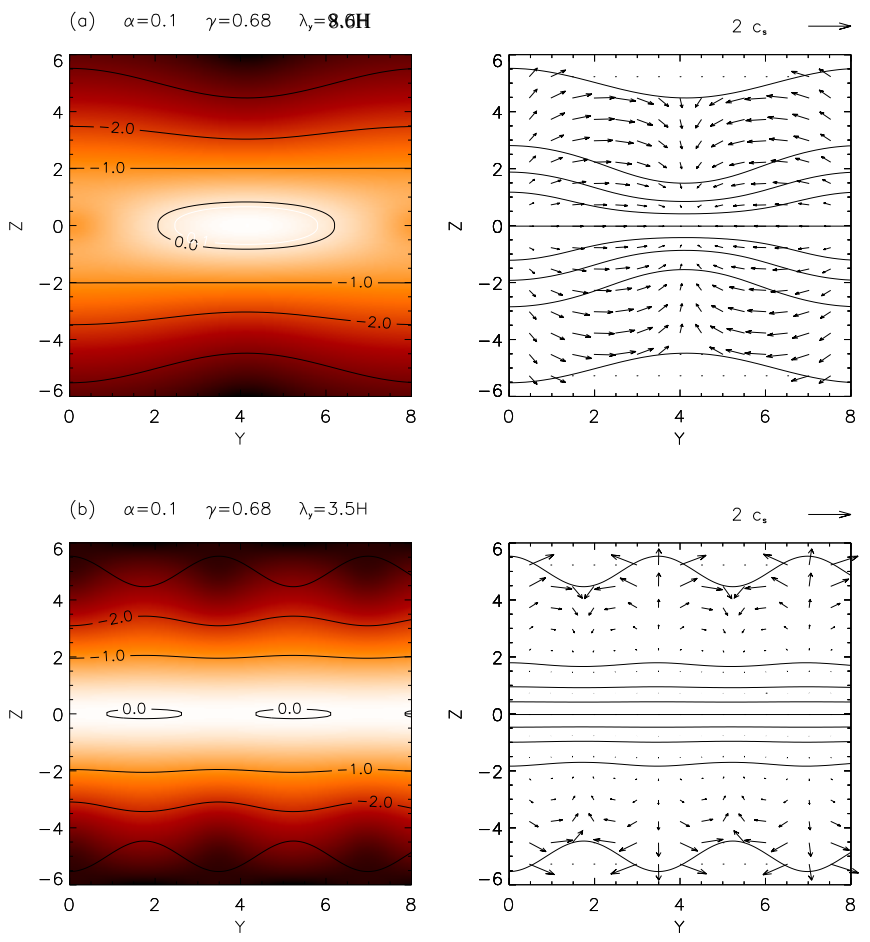

(c) $\alpha=0.1 \quad \gamma=0.68 \quad \lambda_{y}=8.6 \mathrm{H}$
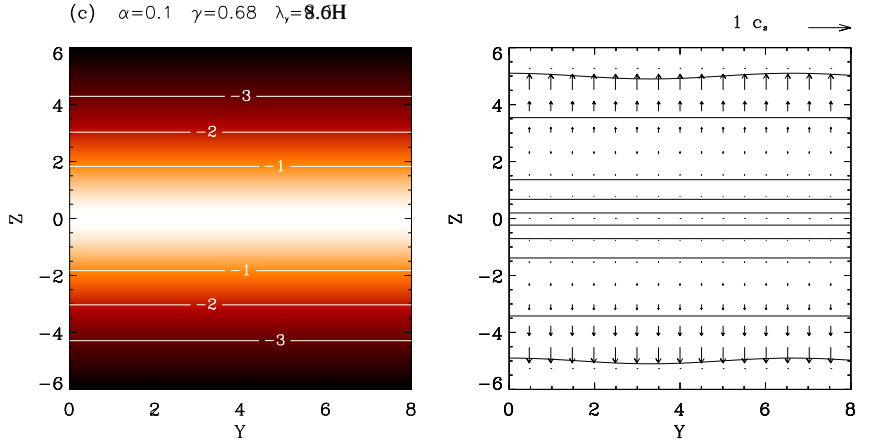

Fig. 4.- Eigen-solutions of the undular mode with odd-parity $(a, b)$ and with even-parity (c). Disk and perturbation parameters are specified on top of each panels on the left hand side. The abscissa and ordinate are $y$ and $z$ coordinates, respectively. The left hand side panels illustrate the density field, and color changes from dark red to white as logarithm of $\rho_{1}(y, z) / \rho_{\mathrm{o}}(z=0)$ increases. Arrow vectors in the right hand side panels illustrate the velocity field with magnitude reference being given on top of each frame. The thin solid lines represent the magnetic field lines and the thick lines do the disk-halo boundary. Case $(a)$ is an example of the self-gravity dominating Parker-Jeans instability, while in $(b)$ convection dominates, particularly in high altitudes. In case $(c)$ the gravitational instability is not triggered, because even-parity condition is imposed upon the solution. 


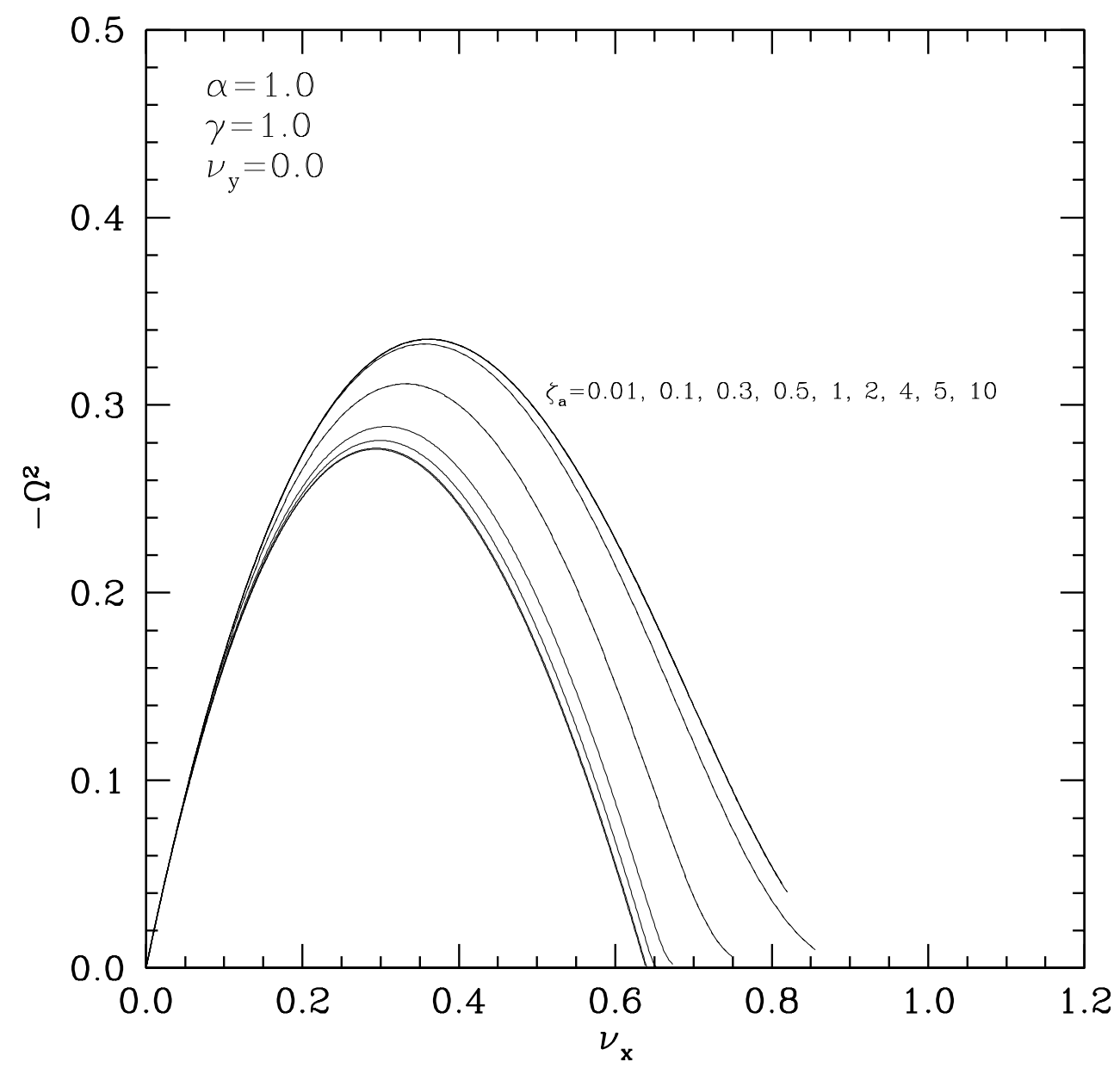

Fig. 5.- Dispersion relation of the interchange mode $\left(\nu_{y}=0\right)$ for various disk thickness. The abscissa denotes effective wave number $\nu_{x}$ and the ordinate does the growth rate squared. To each curve is marked half thickness of the disk, and other disk parameters are given in the upper left corner. 


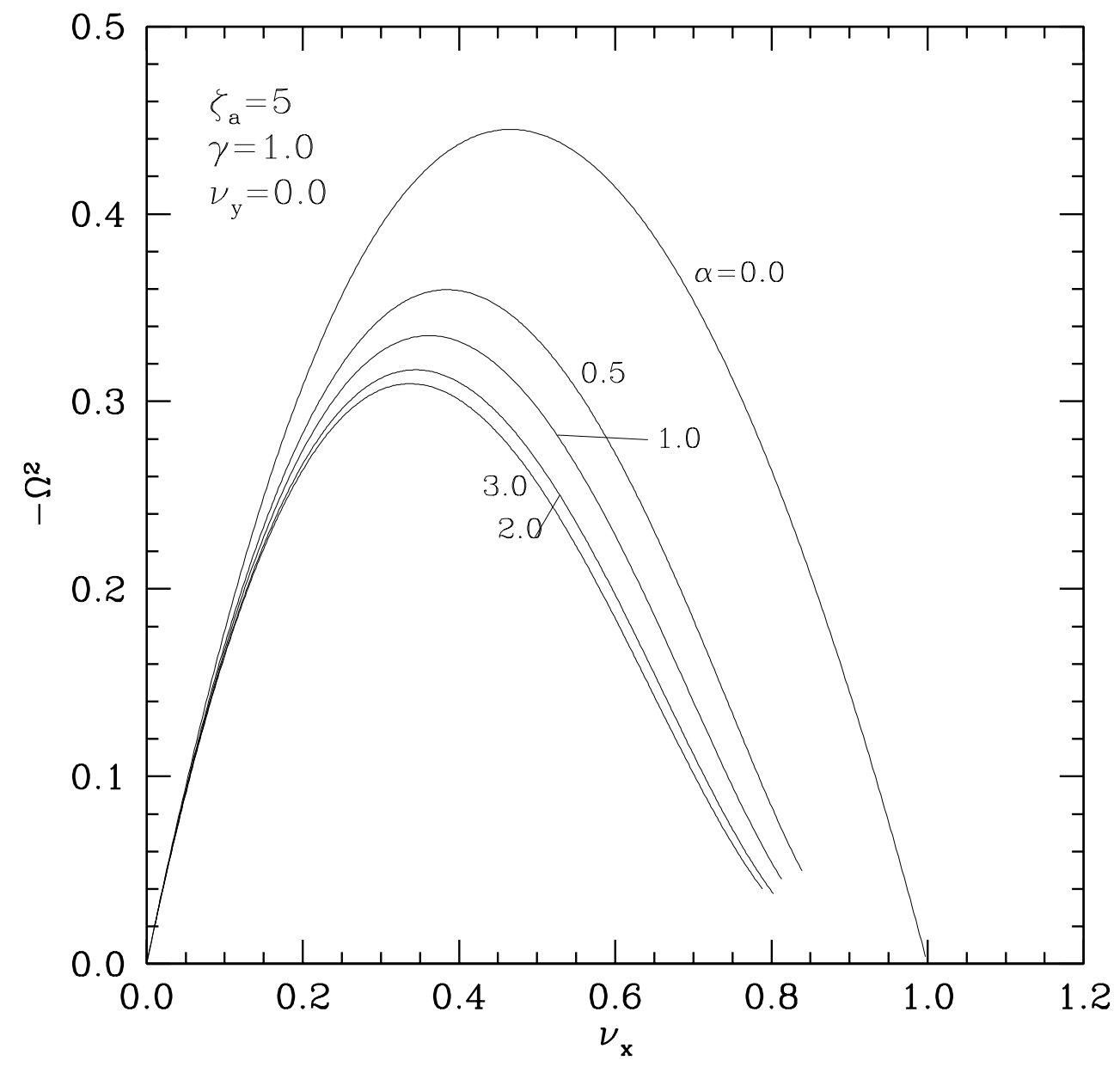

Fig. 6.- Dispersion relation of the interchange mode $\left(\nu_{y}=0\right)$ for a number of selected $\alpha$ 's. The abscissa denotes effective wave number $\nu_{x}$ and the ordinate does the growth rate squared. To each curve is marked $\alpha$ value, and other disk parameters are given in the upper left corner. 


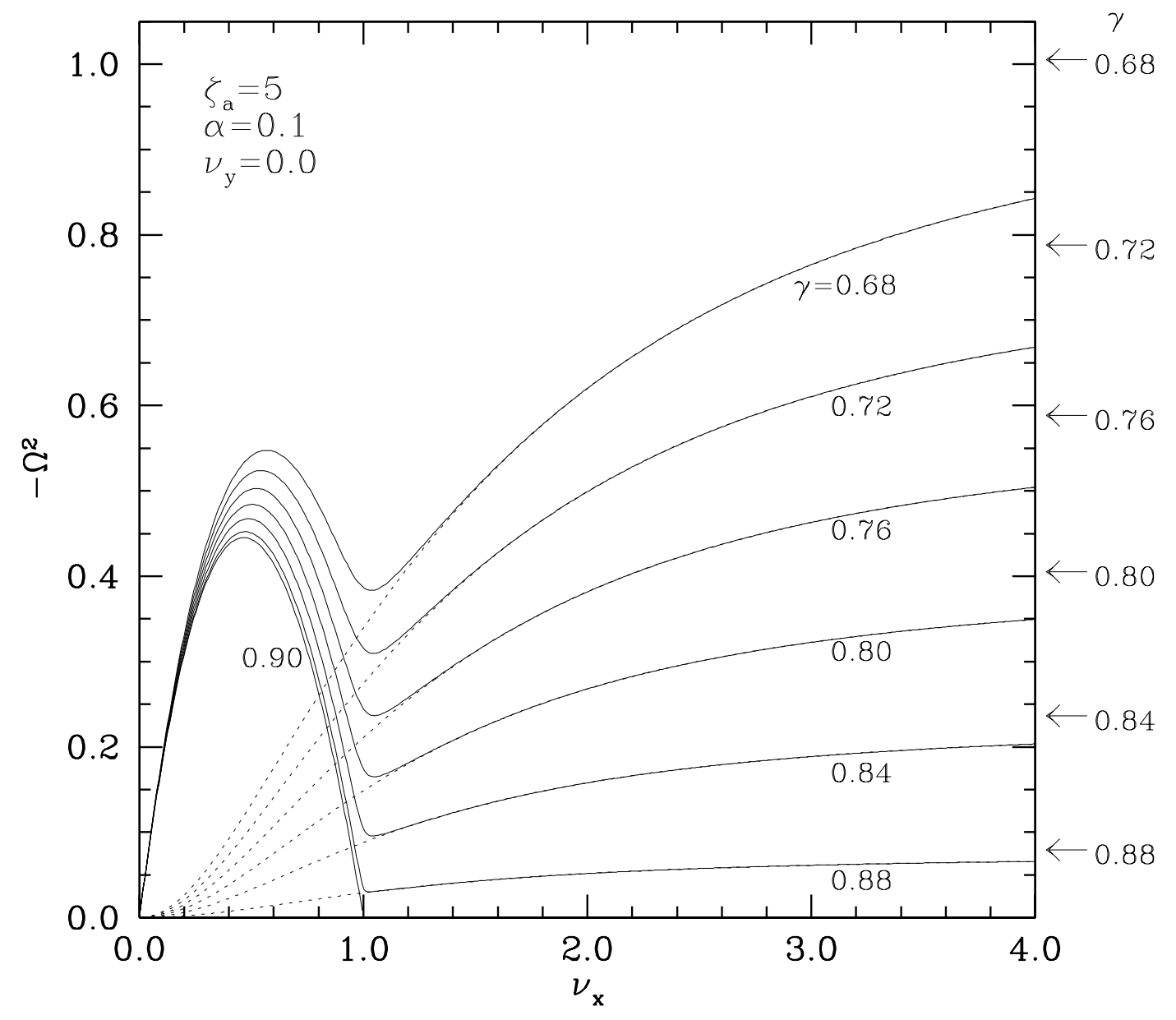

Fig. 7.- Dispersion relation of the interchange mode $(\nu=0)$ for selected $\gamma$ values. The abscissa denotes effective wave number $\nu_{x}$ and the ordinate does the growth rate squared. To each curve $\gamma$ value is marked, and other disk parameters are specified in the upper left corner. The numbers with arrow on the right hand side of the frame indicate square of the maximum growth rate reached in the limit $\nu_{x} \rightarrow \infty$. 
(a) $\quad \alpha=0.1 \quad \gamma=0.68 \quad \lambda_{x}=10.8 H$

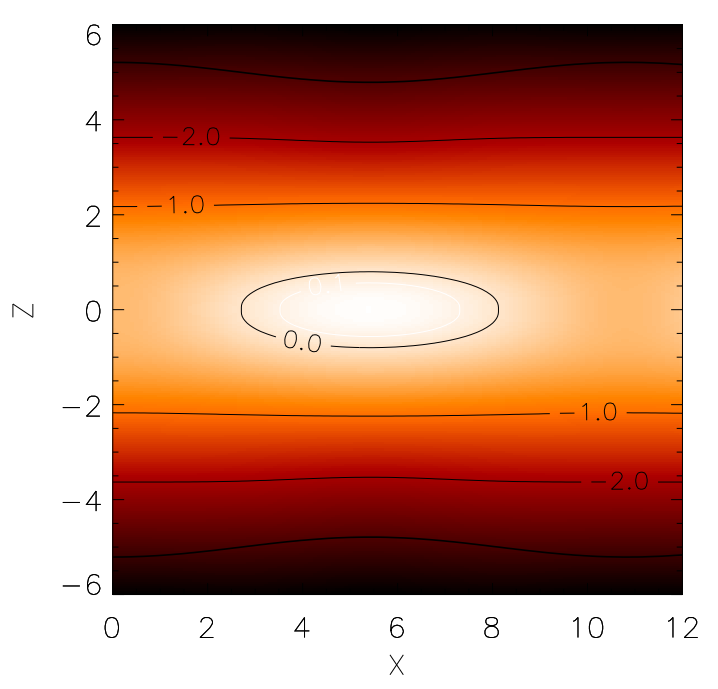

(b) $\quad \alpha=0.1 \quad \gamma=0.68 \quad \lambda_{x}=2.1 \mathrm{H}$

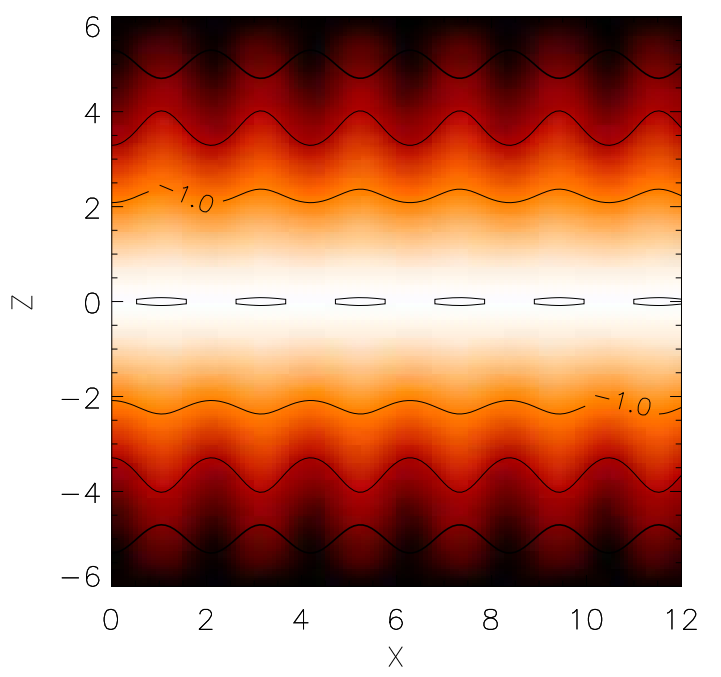

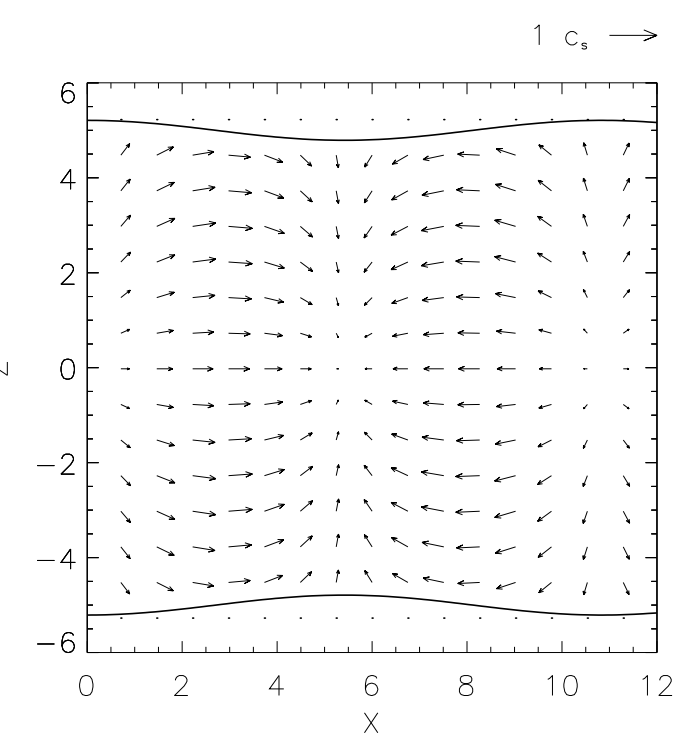

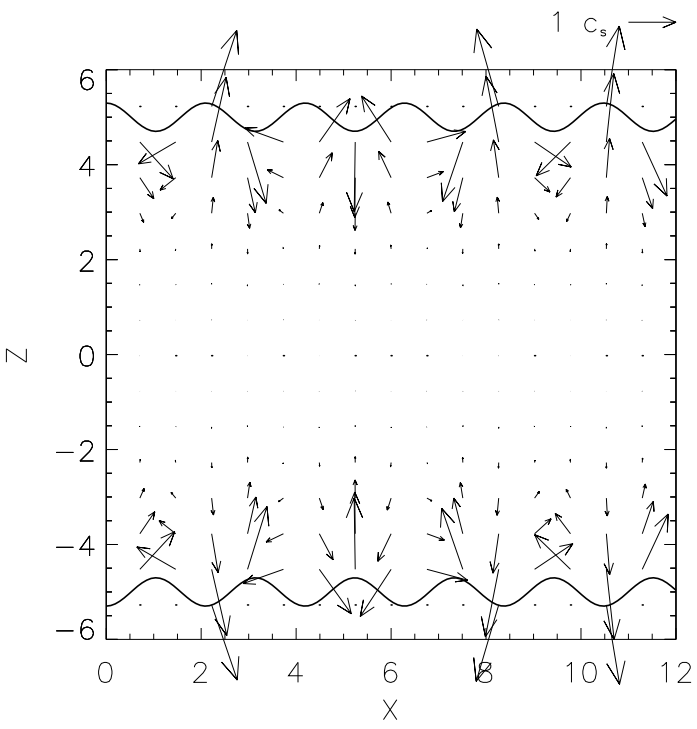

Fig. 8. - Eigen-solutions of the interchange mode with odd-parity $(a)$ and with even-parity (b). Disk and perturbation parameters are specified on top of each panels on the left hand side. The abscissa and ordinate are $x$ and $z$ coordinates, respectively. The left hand side panels illustrate the density field, and color changes from dark red to white as logarithm of $\rho_{1}(y, z) / \rho_{\mathrm{o}}(z=0)$ increases. Arrow vectors in the right hand side panels illustrate the velocity field with magnitude reference being given on top of each frame. The thick lines represent the disk-halo boundary. Case $(a)$ is an example of the Jeans gravitational instability triggered by long wavelength perturbation, while in $(b)$ the convective motions activated by short wavelength perturbation are apparent, particulary in high altitudes. 


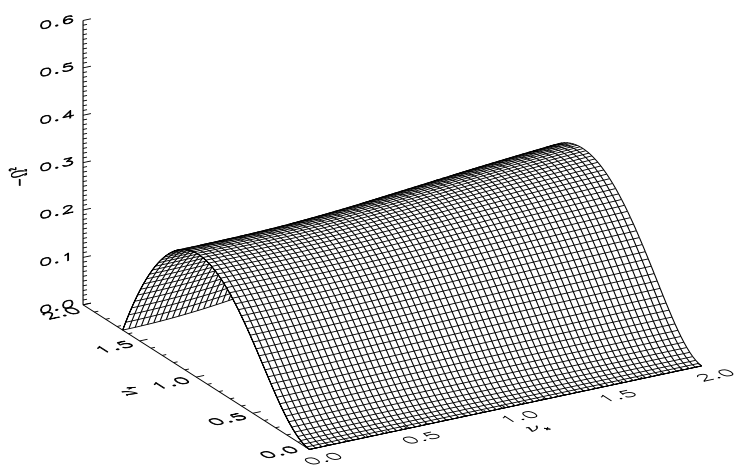

(a)

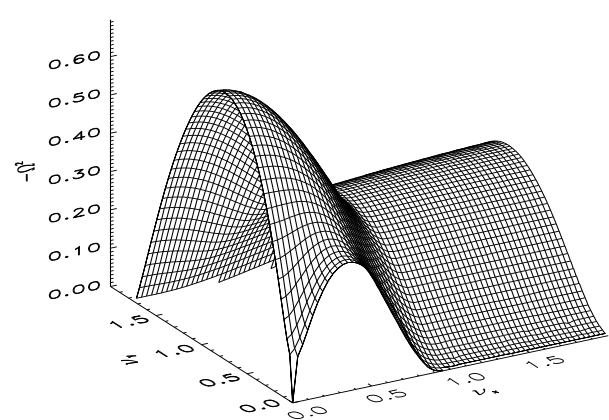

(c)

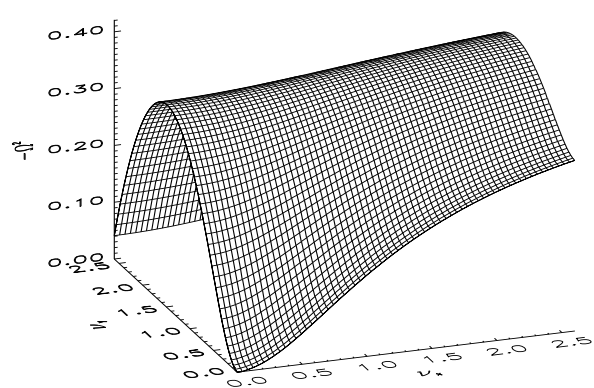

(b)

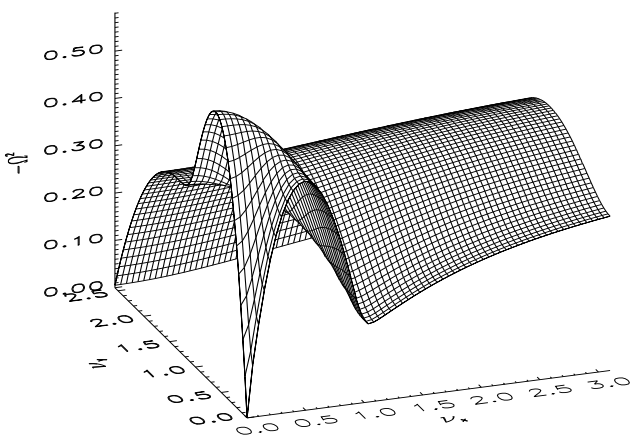

(d)

Fig. 9.- A three-dimensional surface plot for the dispersion relation of the mixed mode. In all four cases the halo is placed at $\zeta_{a}=5$. Other parameters are as follow: In case $(a) \alpha=$ 1 and $\gamma=1$ with even-parity; $(b) \alpha=0.1$ and $\gamma=0.8$ with even-parity; $(c) \alpha=1$ and $\gamma=$ 1 with odd-parity; and $(d) \alpha=0.1$ and $\gamma=0.8$ with odd-parity. The ordinate represents square of the growth rate and the two abscissae denote the horizontal perturbation wave numbers. In $(a)$ and $(b)$ the gravitational instability has not been activated; while in $(c)$ and $(d)$ the Parker-Jeans instability is operative. On the other hand, in $(b)$ and $(d)$, the convection criterion $\gamma<1-\alpha$ for the pure interchange mode is fulfilled, and hence the Rayleigh-Taylor instability gets triggered. The inclining 'ridge' of the growth-rate surface reaches in the $\nu_{x} \rightarrow \infty$ a finite level, which is lower than the Parker-Jeans peak on the $\nu_{y}$ axis. 


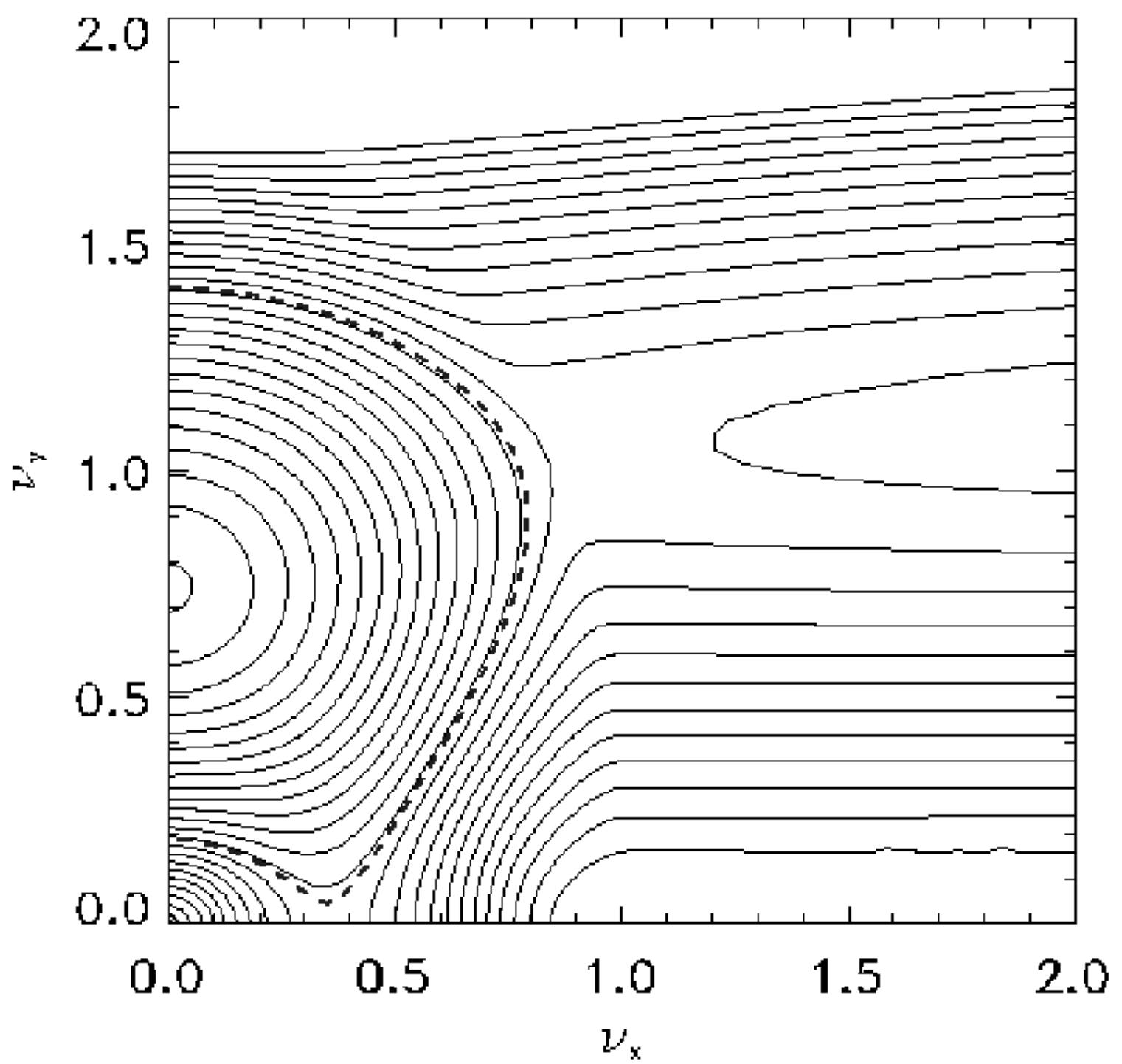

Fig. 10. - Equal growth rate contour map. This is constructed from the 3-dimensional surface plot of the dispersion relation for undular odd-parity solutions with $\zeta_{\mathrm{a}}=5.0, \alpha=1.0$, $\gamma=1.0$. The dashed line contour in blue corresponds to the highest ridge level. Therefore, the perturbations whose wave number pair falls within the blue contour are expected to grow faster than the convective instability. 


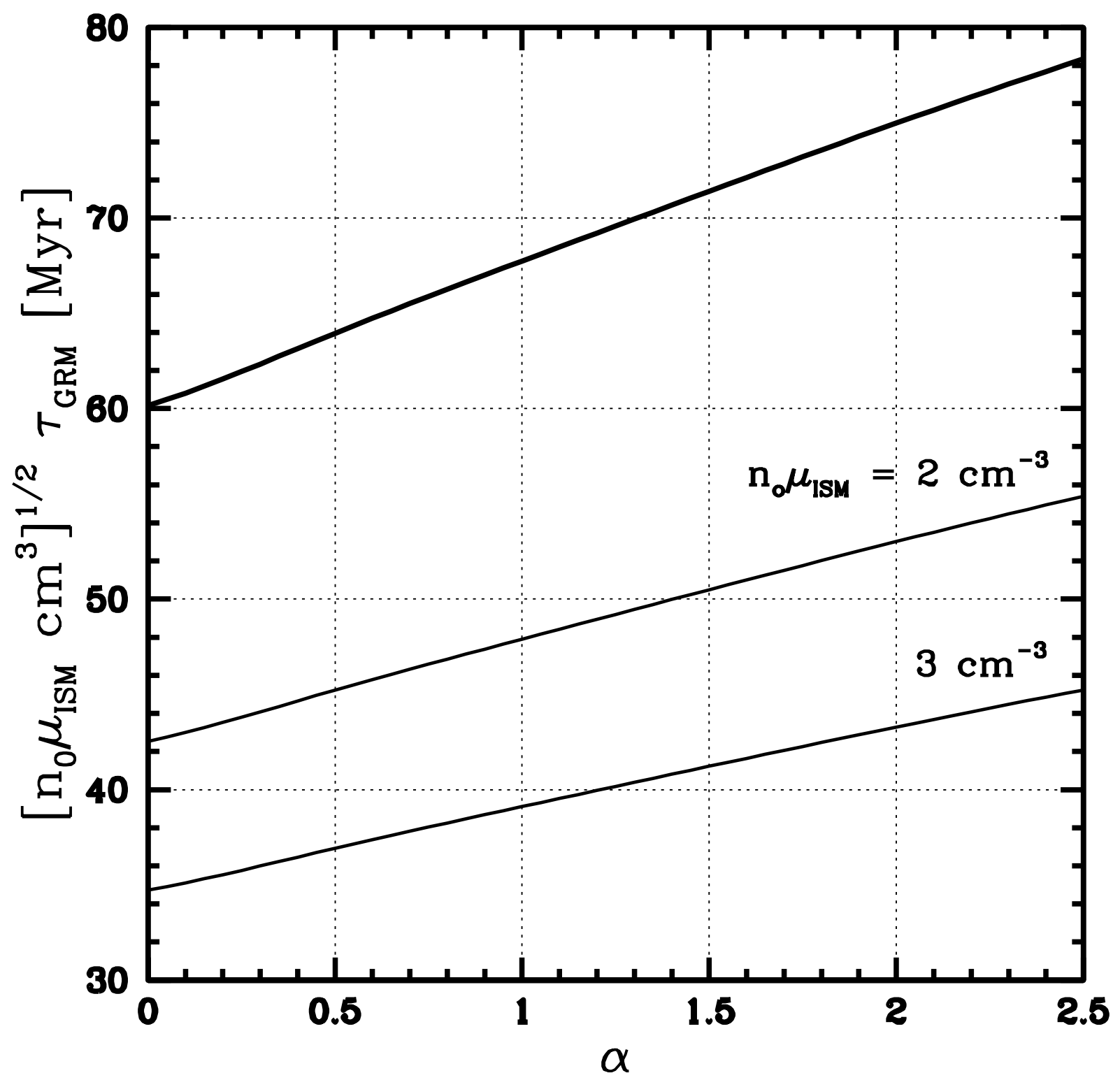

Fig. 11.- Growth time scale versus magnetic-to-gas pressure ratio. Notice that the $n_{\mathrm{o}} \mu_{\mathrm{ISM}}$ factor is absorbed in the ordinate. When the ordinates for the lower two curves in thin solid line are read directly off the figure, the density factor should be ignored. 


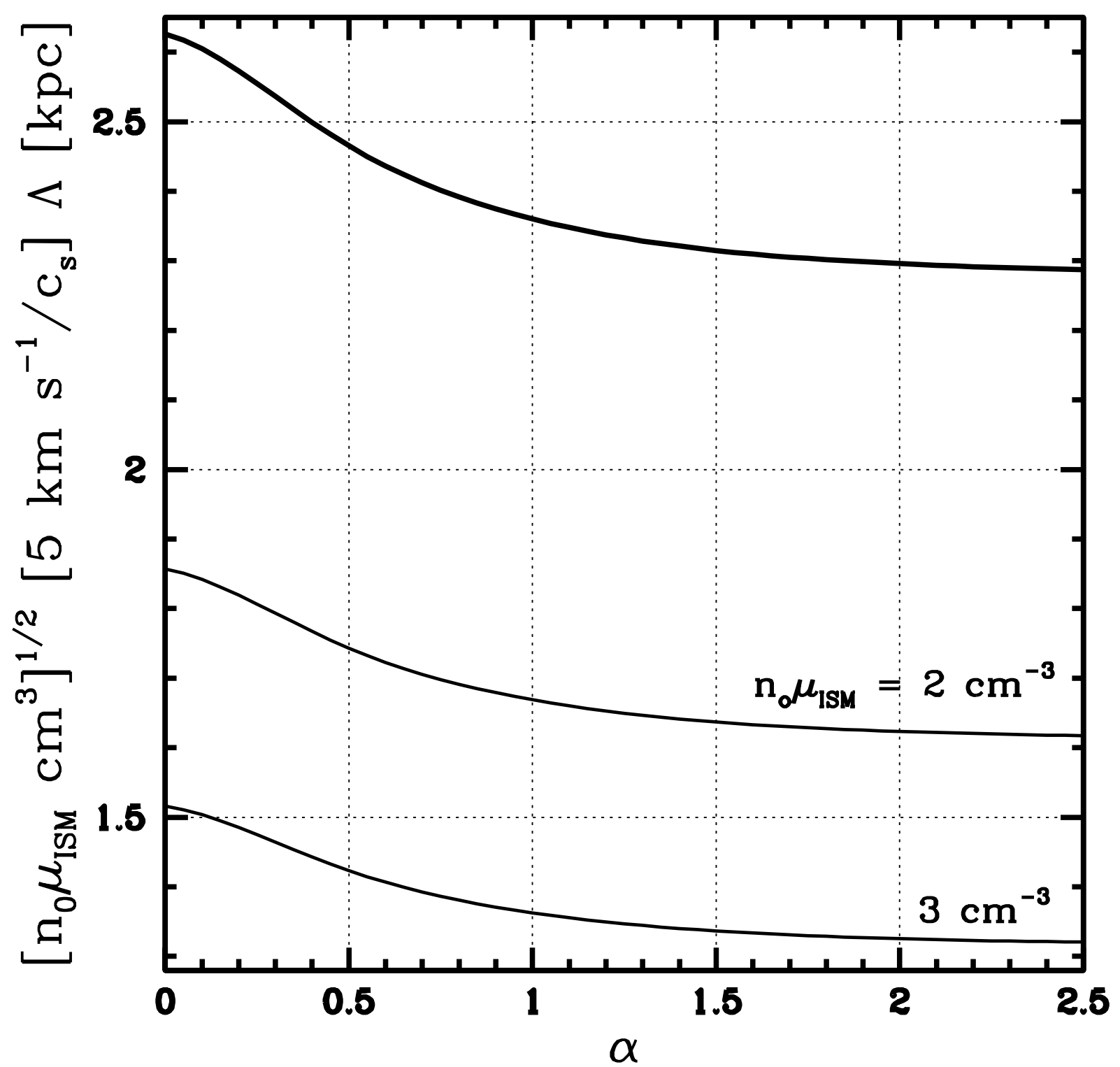

Fig. 12.- Wavelength of maximum growth rate versus magnetic-to-gas pressure ratio. Notice that $n_{\mathrm{o}} \mu_{\mathrm{ISM}}$ and velocity dispersion are absorbed in the ordinate for thick solid line. When the ordinates for the lower two curves in thin solid line are read, the multiple of the two factors should be considered unity. 


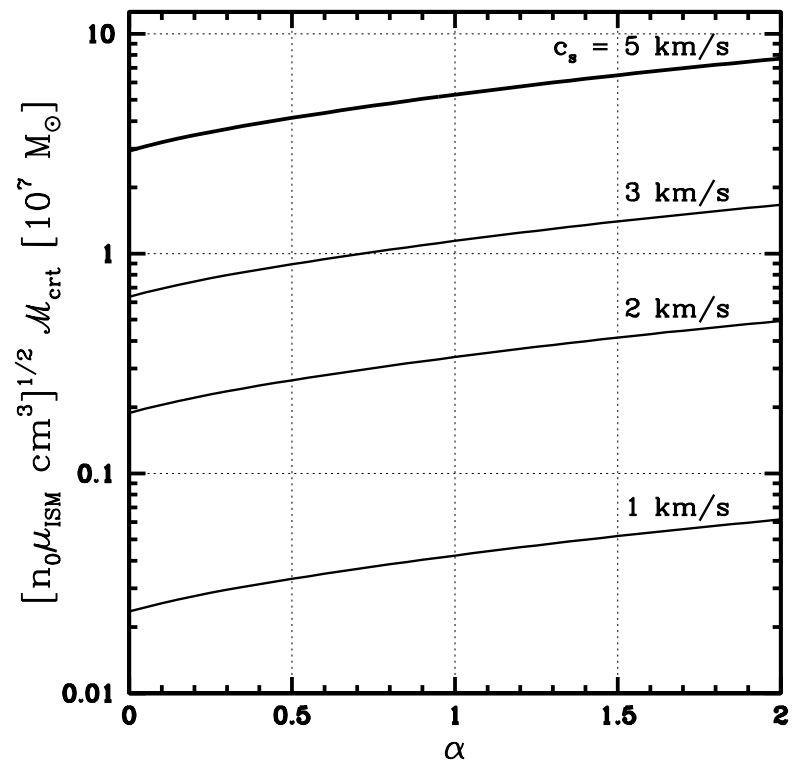

(a)

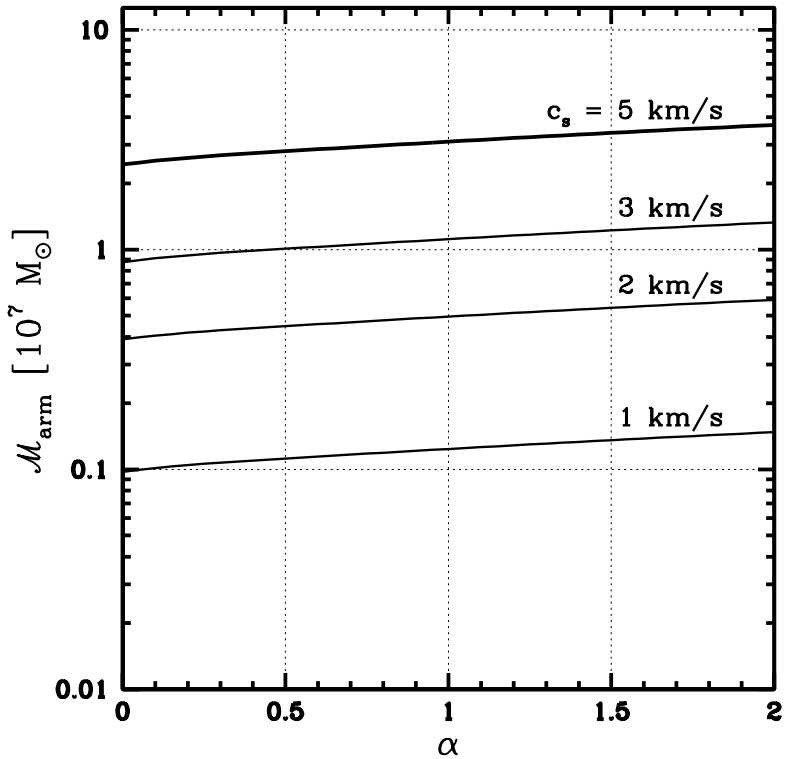

(b)

Fig. 13. - Mass scales based on the critical wave number $(a)$ and the spiral arm width $(b)$ are plotted against magnetic-to-gas pressure ratio for four selected cases of velocity dispersion. Notice that the $n_{\mathrm{o}} \mu_{\mathrm{ISM}}$ factor is absorbed in the ordinate of $(a)$. Once the global dynamics of rotating, self-gravitating, magnetized, gaseous disk fixes the arm width for us, the mass scale based on the arm width doesn't depend on the midplane density explicitly; it does on velocity dispersion to the second power. To prepare this figure $\Delta R=1 \mathrm{kpc}$ is used. 


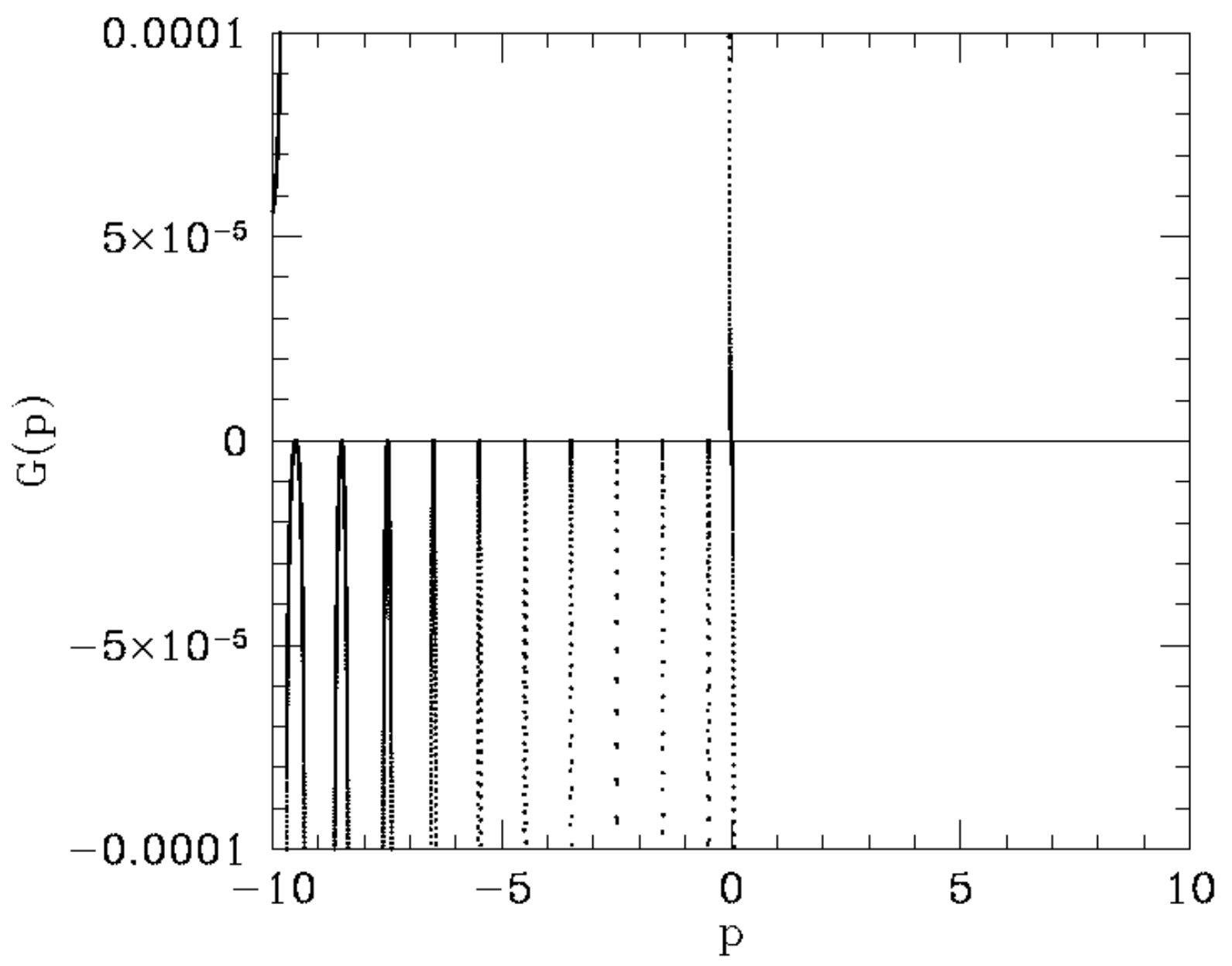

Fig. A1. $-G(p)$ versus $p$ plot. The secular equation, B10, has zero solutions, where $p$ takes negative half integers including zero. 


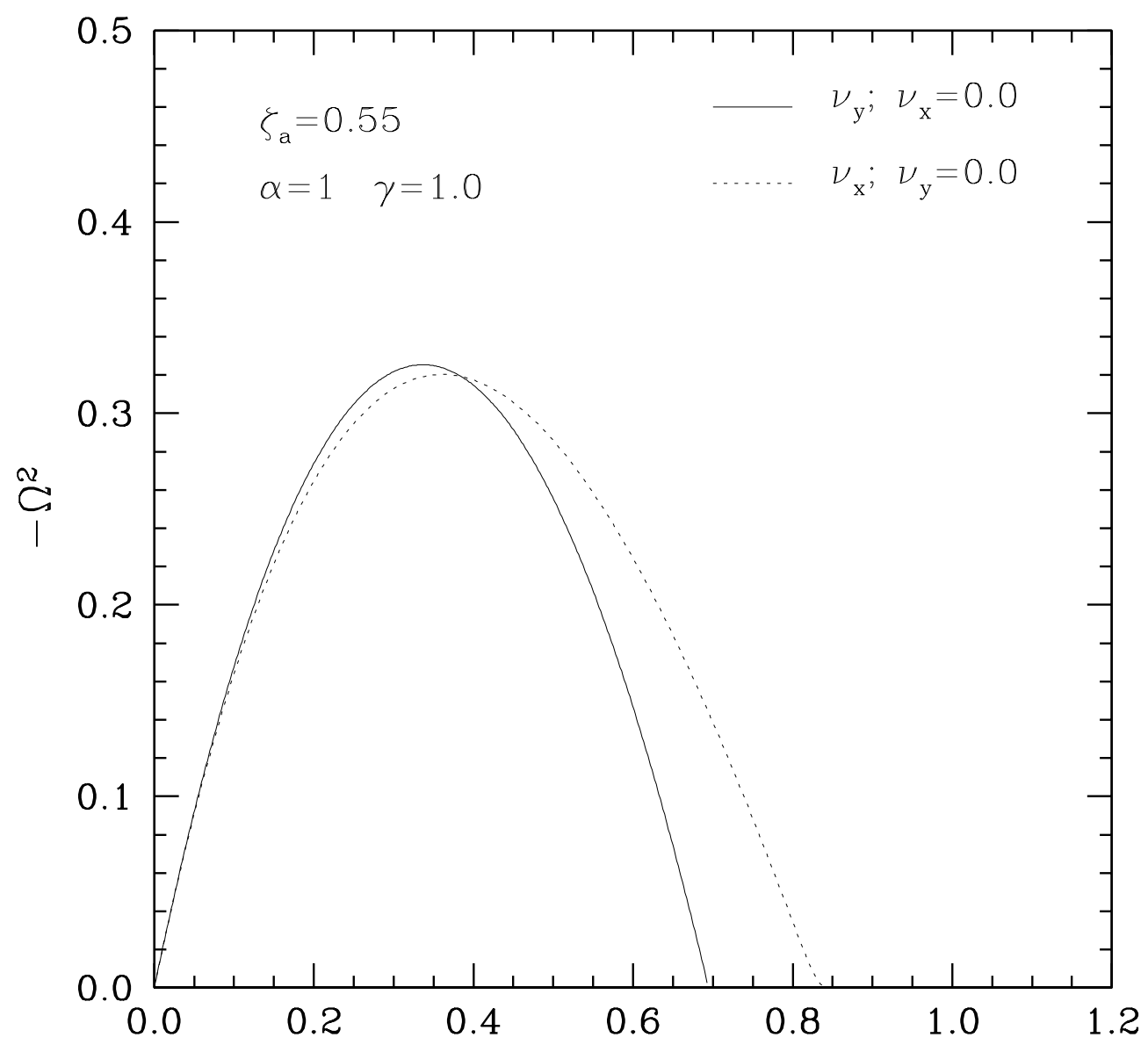

Fig. A2.- Dispersion relations of the critically thick disk. The abscissa represents the normalized wave number $\nu_{y}$ for the undular mode (solid line), and $\nu_{x}$ for the interchange mode (dotted); while the ordinate denotes square of the normalized growth rate. Some of the parameters used in the calculation are given in the upper left corner of the frame. 